Acta Crystallographica Section B

Structural

Science

ISSN 0108-7681

Constantinos D. Antoniadis, ${ }^{a}$ Alexander J. Blake, ${ }^{\mathbf{b}_{*}}$ Sotiris K. Hadjikakou, ${ }^{a}$ Nick Hadjiliadis, Peter Hubberstey, ${ }^{b}$ Martin Schröder $^{b}$ and Claire Wilson ${ }^{b}$

${ }^{a}$ Section of Inorganic and Analytical Chemistry, Department of Chemistry, University of Ioannina, 45110 loannina, Greece, and ${ }^{\mathbf{b}}$ School of Chemistry, The University of Nottingham, University Park, Nottingham NG7 2RD, England

Correspondence e-mail: a.j.blake@nottingham.ac.uk

\title{
Structural characterization of selenium and selenium-diiodine analogues of the antithyroid drug 6-n-propyl-2-thiouracil and its alkyl derivatives
}

The structures of four selenium analogues of the antithyroid drug 6- $n$-propyl-2-thiouracil [systematic name: 2,3 -dihydro-6$n$-propyl-2-thioxopyrimidin-4(1H)-one], namely 6-methyl-2selenouracil, $\quad \mathrm{C}_{5} \mathrm{H}_{6} \mathrm{~N}_{2} \mathrm{OSe} \quad$ (1), 6-ethyl-2-selenouracil, $\mathrm{C}_{6} \mathrm{H}_{8} \mathrm{~N}_{2} \mathrm{OSe}$ (2), 6- $n$-propyl-2-selenouracil, $\mathrm{C}_{7} \mathrm{H}_{10} \mathrm{~N}_{2} \mathrm{OSe}$ (3), and 6-isopropyl-2-selenouracil, $\mathrm{C}_{7} \mathrm{H}_{10} \mathrm{~N}_{2} \mathrm{OSe}$ (4), are described, along with that of the dichloromethane monosolvate of 6-isopropyl-2-selenouracil, $\mathrm{C}_{7} \mathrm{H}_{10} \mathrm{~N}_{2} \mathrm{OSe} \cdot \mathrm{CH}_{2} \mathrm{Cl}_{2}$ $\left(4 \cdot \mathrm{CH}_{2} \mathrm{Cl}_{2}\right)$. The extended structure of (1) is a two-dimensional sheet of topology $6^{3}$ with a brick-wall architecture. The extended structures of (2) and (4) are analogous, being based on a chain of eight-membered $R_{8}^{6}(32)$ hydrogen-bonded rings. In (3) and $\left(4 \cdot \mathrm{CH}_{2} \mathrm{Cl}_{2}\right), R_{2}^{2}(8)$ hydrogen bonding links molecules into chains. 6- $n$-Propyl-2-selenouracil $\cdot \mathrm{I}_{2}$, $\mathrm{C}_{7} \mathrm{H}_{10} \mathrm{~N}_{2} \mathrm{OSe} \cdot \mathrm{I}_{2}$ (7), is a charge-transfer complex with a 'spoke' structure, the extended structure of which is based on a linear chain formed principally by intermolecular $\mathrm{N}-$ $\mathrm{H}$. . O hydrogen bonds. Re-crystallization of 6-ethyl-2-selenouracil or (7) from acetone gave crystals of the diselenides $\quad\left[N\right.$ - $\left(6^{\prime}\right.$-ethyl-4'-pyrimidone $)(6$-ethyl-2-selenouracil $\left.)_{2}(\mathrm{Se}-\mathrm{Se})\right] \cdot 2 \mathrm{H}_{2} \mathrm{O} \quad\left(9 \cdot 2 \mathrm{H}_{2} \mathrm{O}\right)$ or $\left[N-\left(6^{\prime}-n\right.\right.$-propyl-4'-pyrimidone)(6- $n$-propyl-2-selenouracil $\left.)_{2}(\mathrm{Se}-\mathrm{Se})\right](10)$, respectively: these have similar extended chain structures formed via $\mathrm{N}-$ $\mathrm{H} \cdots \mathrm{O}$ and $\mathrm{C}-\mathrm{H} \cdots \mathrm{O}$ hydrogen bonds, stacked to give twodimensional sheets. Re-crystallization of (7) from methanol/ acetonitrile led via deselenation to the formation of crystals of 6- $n$-propyl-2-uracil (11), in which six symmetry-related molecules combine to form a six-membered $R_{6}^{6}(24)$ hydrogen-bonded ring, with each pair of molecules linked by an $R_{2}^{2}(8)$ motif.

\section{Introduction}

Prior to 1960, no uncharged covalent compounds between selenium and iodine had been discovered (Dasent, 1965). It was during the decade 1960-1969 that the structures of the first selenoether-iodine complexes were first reported (Chao \& McCullough, 1961; Hope \& McCullough, 1962; Maddox \& McCullough, 1966; Bjorvatten, 1963; Dahl \& Hassel, 1965; Holmesland \& Römming, 1966; Bent, 1968). Currently, organic selones and/or selenoamides are recognized as potential donors towards diiodine and some iodine-containing compounds, generating charge-transfer complexes that are generally more stable than those of the corresponding sulfur ligands (e.g. du Mont et al., 2001). The reactions between selones or selenoamides with diiodine $\mathrm{I}_{2}$ or interhalogens $\mathrm{I}-$ $X(X=\mathrm{Br}$ or $\mathrm{Cl})$ lead to the formation of iodine chargetransfer complexes (Aragoni et al., 1999, and references therein), which adopt a number of structures including:
Received 8 March 2006 Accepted 29 March 2006
(C) 2006 International Union of Crystallography Printed in Great Britain - all rights reserved 
(i) 'spoke structures' or 'extended spoke structures' bearing a linear arrangement of $\mathrm{Se}-\mathrm{I}-X(X=\mathrm{I}, \mathrm{Br}$ or $\mathrm{Cl})$ or $\mathrm{Se}-\mathrm{I}-$ I . . I-I groups;

(ii) two-coordinate iodine(I) cationic or iodonium salts with selone ligands $([L \mathrm{Se}-X-\mathrm{Se} L])$;

(iii) donor oxidation products including dicationic diselenides $[L \mathrm{Se}-\mathrm{Se} L]^{2+} \cdot 2 \mathrm{I}_{3}{ }^{-}$or neutral diselenides; and

(iv) T-shaped compounds containing the linear group I$\mathrm{Se}-X(X=\mathrm{I}, \mathrm{Br}$ or $\mathrm{Cl}$; Aragoni et al., 2001, and references therein).

The mono-cationic diselenide geometry is the only interaction not yet observed in products of reactions between iodine and selones. As part of a study of charge-transfer complexes with iodine and polyiodides (Blake et al., 1995; Blake, Devillanova, Garau et al., 1998; Blake, Devillanova, Gould et al., 1998; Blake, Li et al., 1998), we report herein the structures of 6- $n$ propyl-2-selenouracil $\cdot \mathrm{I}_{2}, 6$ - $n$-propyl-2-selenouracil itself, some selenium analogues of 6-alkyl-2-thiouracils, two diselenides and the deselenation product 6 - $n$-propyl-2-uracil.

\section{Experimental}

\subsection{Synthesis and crystal growth}

The preparative details for all nine compounds [see Scheme (I)] have been reported previously (Antoniadis et al., 2006). Compounds (1), (2), (3) and (4) were re-crystallized from water to give colourless crystals, while re-crystallization of (4)

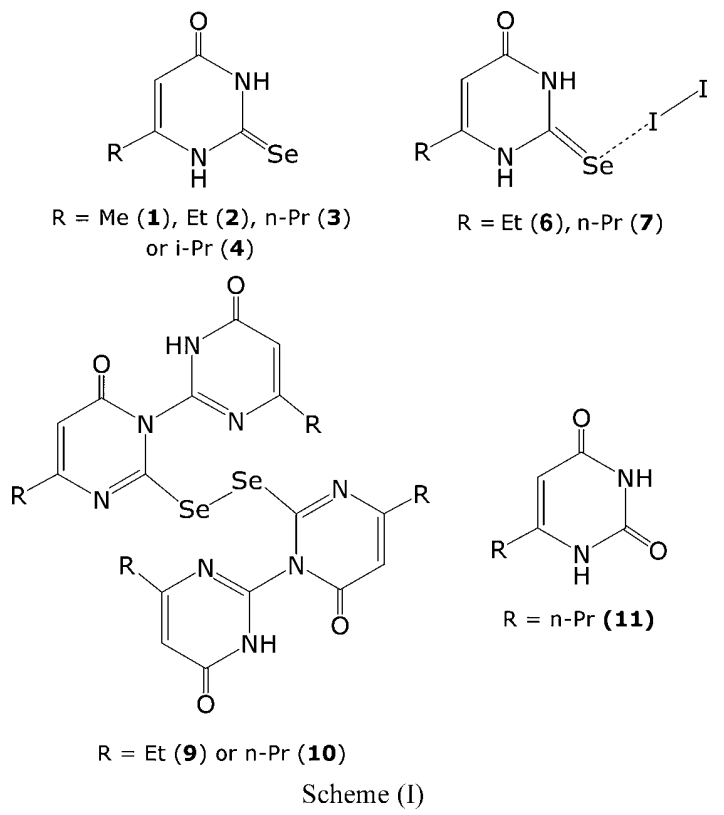

from dichloromethane gave crystals of $\left(4 \cdot \mathrm{CH}_{2} \mathrm{Cl}_{2}\right)$. Crystals of (7) were grown from chloroform solutions, while re-crystallization of (6) and (7) from acetone gave the oxidation products $\left(9 \cdot 2 \mathrm{H}_{2} \mathrm{O}\right)$ and (10), respectively. Re-crystallization of (7) from methanol/acetonitrile led via deselenation to crystals of 6- $n$-propyl-2-uracil (11).

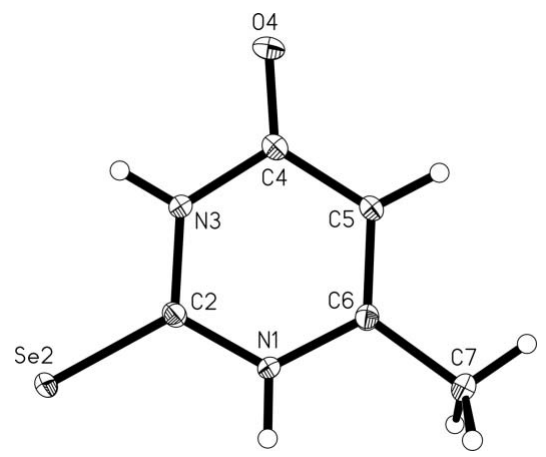

Figure 1

Displacement ellipsoid plot of (1) showing the atom-numbering scheme, with ellipsoids drawn at the $50 \%$ probability level.

\subsection{Data collection, structure solution and refinement}

Details of cell data, data collection and structure solution and refinement are summarized in Table $1^{\mathbf{1}}$. Except for $\left(9 \cdot 2 \mathrm{H}_{2} \mathrm{O}\right)$, which was solved using SIR92 direct methods (Altomare et al., 1994), the structures were solved by direct methods using SHELXS97 (Sheldrick, 1990) and developed by difference Fourier methods using SHELXL97 (Sheldrick, 1998). With the exception of the $s p^{2}$-bound methyl group in (1), where they were located from a circular difference Fourier synthesis and refined as part of a rigid rotating group, all carbon- and nitrogen-bound $\mathrm{H}$ atoms were placed in geometrically calculated positions and refined using riding models (SHELXL97; Sheldrick, 1998). Crystals of $\left(9 \cdot 2 \mathrm{H}_{2} \mathrm{O}\right)$ are affected by twinning, principally by a $180^{\circ}$ rotation about [001] for which the twin fraction was 0.291 (2). The water $\mathrm{H}$ atoms in $\left(9 \cdot 2 \mathrm{H}_{2} \mathrm{O}\right)$ were not located.

The structure determination of (3) posed particular difficulties: the crystals were very small in two dimensions (10 and $20 \mu \mathrm{m}$ ) and efforts to obtain a dataset using a sealed X-ray tube or a rotating-anode source gave no significant diffraction. However, we were able to obtain a weak dataset on Station 9.8 of the Daresbury Synchrotron Radiation Source, although even this did not overcome all of the limitations imposed by the poor crystal quality. We found that only the Se atoms could be refined with anisotropic displacement parameters, and a total of 99 geometric, planarity and similarity restraints were necessary for a stable refinement. Although we cannot therefore discuss the fine details of the molecular geometry, it did prove possible to obtain reliable information about the extended structure of (3).

\section{Results and discussion}

\subsection{Molecular structures of (1)-(4), $\left(4 \cdot \mathrm{CH}_{2} \mathrm{Cl}_{2}\right)$ and (11)}

Displacement ellipsoid plots of (1)-(4), (4. $\left.\mathrm{CH}_{2} \mathrm{Cl}_{2}\right)$ and (11) are shown in Figs. 1-6 while selected interatomic distances and angles are listed in Tables 2 and 4. Although the asymmetric

\footnotetext{
${ }^{1}$ Supplementary data for this paper are available from the IUCr electronic archives (Reference: SO5003). Services for accessing these data are described at the back of the journal.
} 
Table 1

Experimental table.

\begin{tabular}{|c|c|c|c|c|c|}
\hline & (1) & (2) & (3) & (4) & $\left(4 \cdot \mathrm{CH}_{2} \mathrm{Cl}_{2}\right)$ \\
\hline \multicolumn{6}{|l|}{ Crystal data } \\
\hline Chemical formula & $\mathrm{C}_{5} \mathrm{H}_{6} \mathrm{~N}_{2} \mathrm{OSe}$ & $\mathrm{C}_{6} \mathrm{H}_{8} \mathrm{~N}_{2} \mathrm{OSe}$ & $\mathrm{C}_{7} \mathrm{H}_{10} \mathrm{~N}_{2} \mathrm{OSe}$ & $\mathrm{C}_{7} \mathrm{H}_{10} \mathrm{~N}_{2} \mathrm{OSe}$ & $\mathrm{C}_{8} \mathrm{H}_{12} \mathrm{Cl}_{2} \mathrm{~N}_{2} \mathrm{OSe}$ \\
\hline$M_{r}$ & 189.08 & 203.10 & 217.13 & 217.13 & 302.06 \\
\hline $\begin{array}{l}\text { Cell setting, space } \\
\text { group }\end{array}$ & Monoclinic, $P 2_{1} / c$ & Triclinic, $P \overline{1}$ & Orthorhombic, Pbca & Triclinic, $P \overline{1}$ & Triclinic, $P \overline{1}$ \\
\hline Temperature (K) & $120(2)$ & $150(2)$ & $120(2)$ & $150(2)$ & $150(2)$ \\
\hline$a, b, c(\AA)$ & $\begin{array}{l}4.3411(7), 14.756(2) \\
\quad 9.690(2)\end{array}$ & $\begin{array}{l}8.394(2), 10.029(2), \\
\quad 14.931(4)\end{array}$ & $\begin{array}{l}10.568(7), 11.257(7) \\
28.79(2)\end{array}$ & $\begin{array}{l}8.9192(9), \\
10.6403(10), \\
15.1965(15)\end{array}$ & $\begin{array}{l}8.841(3), 11.259(3), \\
\quad 12.424(4)\end{array}$ \\
\hline$\alpha, \beta, \gamma\left(^{\circ}\right)$ & $90.00,90.157$ (2), 90.00 & $\begin{array}{c}101.023(4), \\
100.893(4), \\
105.705(4)\end{array}$ & $90.00,90.00,90.00$ & $\begin{array}{l}106.019(2), \\
105.366(2) \\
96.166(2)\end{array}$ & $\begin{array}{l}90.450(5), 105.350(4) \\
\quad 92.945(5)\end{array}$ \\
\hline$V\left(\AA^{3}\right)$ & $620.71(18)$ & $1148.5(5)$ & $3425(4)$ & $1311.0(2)$ & $1190.7(6)$ \\
\hline$Z$ & 4 & 6 & 16 & 6 & 4 \\
\hline$D_{x}\left(\mathrm{Mg} \mathrm{m}^{-3}\right)$ & 2.023 & 1.762 & 1.684 & 1.650 & 1.685 \\
\hline Radiation type & Synchrotron & Mo $K \alpha$ & Synchrotron & Мo $K \alpha$ & Мо $K \alpha$ \\
\hline $\begin{array}{l}\text { No. of reflections for } \\
\text { cell parameters }\end{array}$ & 4328 & 2232 & 3142 & 3689 & 2249 \\
\hline$\theta$ range $\left({ }^{\circ}\right)$ & $2.6-29.0$ & $2.6-24.8$ & $2.6-27.5$ & $2.4-27.6$ & $2.4-24.6$ \\
\hline$\mu\left(\mathrm{mm}^{-1}\right)$ & 5.96 & 4.84 & 4.33 & 4.24 & 3.57 \\
\hline Crystal form, colour & Needle, colourless & $\begin{array}{l}\text { Triangular prism, } \\
\text { colourless }\end{array}$ & Lath, colourless & Tablet, colourless & Plate, colourless \\
\hline Crystal size (mm) & $0.10 \times 0.01 \times 0.01$ & $0.21 \times 0.12 \times 0.04$ & $0.20 \times 0.02 \times 0.01$ & $0.25 \times 0.14 \times 0.06$ & $0.36 \times 0.22 \times 0.02$ \\
\hline Data collection & & & & & \\
\hline Diffractometer & $\begin{array}{l}\text { Bruker SMART } \\
\text { APEXII CCD } \\
\text { diffractometer }\end{array}$ & $\begin{array}{l}\text { Bruker SMART APEX } \\
\text { CCD area detector }\end{array}$ & $\begin{array}{l}\text { Bruker SMART } \\
\text { APEXII CCD } \\
\text { diffractometer }\end{array}$ & $\begin{array}{c}\text { Bruker SMART APEX } \\
\text { CCD area detector }\end{array}$ & $\begin{array}{c}\text { Bruker SMART APEX } \\
\text { CCD area detector }\end{array}$ \\
\hline Data collection method & Fine-slice $\omega$ scans & $\omega$ scans & Fine-slice $\omega$ scans & $\omega$ scans & $\omega$ scans \\
\hline Absorption correction & $\begin{array}{l}\text { Multi-scan (based on } \\
\text { symmetry-related } \\
\text { measurements) }\end{array}$ & $\begin{array}{l}\text { Multi-scan (based on } \\
\text { symmetry-related } \\
\text { measurements) }\end{array}$ & $\begin{array}{l}\text { Multi-scan (based on } \\
\text { symmetry-related } \\
\text { measurements) }\end{array}$ & $\begin{array}{l}\text { Multi-scan (based on } \\
\text { symmetry-related } \\
\text { measurements) }\end{array}$ & $\begin{array}{l}\text { Multi-scan (based on } \\
\text { symmetry-related } \\
\text { measurements) }\end{array}$ \\
\hline$T_{\min }$ & 0.816 & 0.687 & 0.260 & 0.581 & 0.684 \\
\hline$T_{\max }$ & 1.000 & 1.000 & 1.000 & 0.770 & 1.000 \\
\hline $\begin{array}{l}\text { No. of measured, } \\
\text { independent and } \\
\text { observed reflections }\end{array}$ & $6350,1770,1642$ & $8174,4025,3189$ & $24855,3471,2417$ & $8163,5780,4932$ & $10743,6193,4391$ \\
\hline $\begin{array}{l}\text { Criterion for observed } \\
\text { reflections }\end{array}$ & $I>2 \sigma(I)$ & $I>2 \sigma(I)$ & $I>2 \sigma(I)$ & $I>2 \sigma(I)$ & $I>2 \sigma(I)$ \\
\hline$R_{\text {int }}$ & 0.027 & 0.038 & 0.250 & 0.013 & 0.050 \\
\hline$\theta_{\max }\left({ }^{\circ}\right)$ & 29.0 & 25.1 & 25.0 & 27.6 & 27.5 \\
\hline Range of $h, k, l$ & $-6 \Rightarrow h \Rightarrow 6$ & $-10 \Rightarrow h \Rightarrow 10$ & $-13 \Rightarrow h \Rightarrow 13$ & $-11 \Rightarrow h \Rightarrow 7$ & $-11 \Rightarrow h \Rightarrow 11$ \\
\hline & $-20 \Rightarrow k \Rightarrow 21$ & $-11 \Rightarrow k \Rightarrow 11$ & $-14 \Rightarrow k \Rightarrow 14$ & $-13 \Rightarrow k \Rightarrow 13$ & $-14 \Rightarrow k \Rightarrow 14$ \\
\hline & $-13 \Rightarrow l \Rightarrow 13$ & $-17 \Rightarrow l \Rightarrow 17$ & $-35 \Rightarrow l \Rightarrow 35$ & $-17 \Rightarrow l \Rightarrow 19$ & $-16 \Rightarrow l \Rightarrow 16$ \\
\hline Refinement & & & & & \\
\hline Refinement on & $F^{2}$ & $F^{2}$ & $F^{2}$ & $F^{2}$ & $F^{2}$ \\
\hline $\begin{array}{l}R\left[F^{2}>2 \sigma\left(F^{2}\right)\right] \\
\quad w R\left(F^{2}\right), S\end{array}$ & $0.025,0.074,0.80$ & $0.056,0.146,1.03$ & $0.173,0.408,1.18$ & $0.028,0.071,1.04$ & $0.052,0.099,0.97$ \\
\hline No. of reflections & 1770 & 4025 & 3471 & 5780 & 6193 \\
\hline No. of parameters & 83 & 271 & 99 & 298 & 254 \\
\hline $\mathrm{H}$-atom treatment & $\begin{array}{l}\text { Rigid rotating group; } \\
\text { riding model }\end{array}$ & $\begin{array}{l}\text { Constrained to parent } \\
\text { site }\end{array}$ & $\begin{array}{l}\text { Constrained to parent } \\
\text { site }\end{array}$ & $\begin{array}{l}\text { Constrained to parent } \\
\text { site }\end{array}$ & $\begin{array}{l}\text { Constrained to parent } \\
\text { site }\end{array}$ \\
\hline Weighting scheme & $\begin{array}{l}w=1 /\left[\sigma^{2}\left(F_{o}^{2}\right)+\right. \\
\left.\quad(0.079 P)^{2}\right], \text { where } \\
P=\left(F_{o}^{2}+2 F_{c}^{2}\right) / 3\end{array}$ & $\begin{array}{c}w=1 /\left[\sigma^{2}\left(F_{o}^{2}\right)+\right. \\
\left.(0.094 P)^{2}\right], \text { where } \\
P=\left(F_{o}^{2}+2 F_{c}^{2}\right) / 3\end{array}$ & $\begin{array}{l}w=1 /\left[\sigma^{2}\left(F_{o}^{2}\right)+\right. \\
\left.\quad(0.025 P)^{2}+367.0\right], \\
\text { where } P=\left(F_{o}^{2}+\right. \\
\left.2 F_{c}^{2}\right) / 3\end{array}$ & $\begin{array}{l}w=1 /\left[\sigma^{2}\left(F_{o}^{2}\right)+\right. \\
\left.\quad(0.033 P)^{2}+0.854 P\right] \\
\text { where } P=\left(F_{o}^{2}+\right. \\
\left.2 F_{c}^{2}\right) / 3\end{array}$ & $\begin{array}{c}w=1 /\left[\sigma^{2}\left(F_{o}^{2}\right)+\right. \\
\left.(0.0305 P)^{2}\right], \text { where } \\
P=\left(F_{o}^{2}+2 F_{c}^{2}\right) / 3\end{array}$ \\
\hline$(\Delta / \sigma)_{\max }$ & 0.001 & 0.001 & 0.002 & 0.001 & 0.001 \\
\hline$\Delta \rho_{\max }, \Delta \rho_{\min }\left(\mathrm{e} \AA^{-3}\right)$ & $0.57,-0.80$ & $1.48,-1.02$ & $2.38,-2.33$ & $0.65,-0.40$ & $0.87,-0.51$ \\
\hline & (7) & (9) & $(10$ & & $(11)$ \\
\hline Crystal data & & & & & \\
\hline Chemical formula & $\mathrm{C}_{7} \mathrm{H}_{10} \mathrm{I}_{2} \mathrm{~N}_{2} \mathrm{OSe}$ & $\mathrm{C}_{24} \mathrm{H}_{26} \mathrm{~N}_{8} \mathrm{O}$ & $\mathrm{e}_{2} \cdot 2 \mathrm{H}_{2} \mathrm{O}$ & ${ }_{34} \mathrm{~N}_{8} \mathrm{O}_{4} \mathrm{Se}_{2}$ & $\mathrm{C}_{7} \mathrm{H}_{10} \mathrm{~N}_{2} \mathrm{O}_{2}$ \\
\hline$M_{r}$ & 470.93 & 684.48 & & & 154.17 \\
\hline $\begin{array}{l}\text { Cell setting, space } \\
\text { group }\end{array}$ & Triclinic, $P \overline{1}$ & Triclinic, $P$ & & nic, $P \overline{1}$ & Rhombohedral, $R \overline{3}$ \\
\hline Temperature (K) & $150(2)$ & $120(2)$ & & & $150(2)$ \\
\hline
\end{tabular}


Table 1 (continued)

\begin{tabular}{|c|c|c|c|c|}
\hline & (7) & $(9)$ & (10) & (11) \\
\hline$a, b, c(\AA)$ & $\begin{array}{l}6.603(2), 7.700(3) \\
\quad 13.037(5)\end{array}$ & $\begin{array}{l}4.8330(2), 9.7970(5) \\
\quad 14.1796(8)\end{array}$ & $\begin{array}{l}5.0717(6), \\
11.8615(14), \\
11.9385(14)\end{array}$ & $\begin{array}{c}19.9444(10), \\
19.9444(10), \\
9.8918(10)\end{array}$ \\
\hline$\alpha, \beta, \gamma\left({ }^{\circ}\right)$ & $\begin{array}{l}75.969(6), 86.808(6), \\
73.113(6)\end{array}$ & $\begin{array}{l}83.490(3), 84.431(3), \\
89.353(3)\end{array}$ & $\begin{array}{l}83.161(2), 82.785(2), \\
\quad 84.358(2)\end{array}$ & $90.00,90.00,120.00$ \\
\hline$V\left(\AA^{3}\right)$ & $615.3(7)$ & $663.91(6)$ & $704.90(14)$ & $3407.6(10)$ \\
\hline$Z$ & 2 & 1 & 1 & 18 \\
\hline$D_{x}\left(\mathrm{Mg} \mathrm{m}^{-3}\right)$ & 2.542 & 1.712 & 1.660 & 1.352 \\
\hline Radiation type & Мо $K \alpha$ & Мo $K \alpha$ & Synchrotron & Mo $K \alpha$ \\
\hline $\begin{array}{l}\text { No. of reflections for } \\
\text { cell parameters }\end{array}$ & 1665 & 2951 & 1672 & 1748 \\
\hline$\theta$ range $\left(^{\circ}\right)$ & $2.8-27.0$ & $2.9-27.5$ & $2.2-27.0$ & $2.4-27.4$ \\
\hline$\mu\left(\mathrm{mm}^{-1}\right)$ & 8.04 & 2.84 & 2.67 & 0.10 \\
\hline Crystal form, colour & Column, red & Needle, orange & Plate, colourless & Lens, orange \\
\hline Crystal size $(\mathrm{mm})$ & $0.22 \times 0.10 \times 0.06$ & $0.20 \times 0.04 \times 0.03$ & $0.08 \times 0.05 \times 0.01$ & $0.52 \times 0.17 \times 0.17$ \\
\hline \multicolumn{5}{|l|}{ Data collection } \\
\hline Diffractometer & $\begin{array}{l}\text { Bruker SMART1000 } \\
\text { CCD area detector }\end{array}$ & $\begin{array}{l}\text { Bruker Nonius } \\
\text { kappaCCD area } \\
\text { detector }\end{array}$ & $\begin{array}{l}\text { Bruker SMART } \\
\text { APEXII CCD } \\
\text { diffractometer }\end{array}$ & $\begin{array}{c}\text { Bruker SMART APEX } \\
\text { CCD area detector }\end{array}$ \\
\hline Data collection method & $\omega$ scans & $\omega$ and $\varphi$ & Fine-slice $\omega$ scans & $\omega$ scans \\
\hline Absorption correction & $\begin{array}{l}\text { Multi-scan (based on } \\
\text { symmetry-related } \\
\text { measurements) }\end{array}$ & $\begin{array}{l}\text { Multi-scan (based on } \\
\text { symmetry-related } \\
\text { measurements) }\end{array}$ & $\begin{array}{l}\text { Multi-scan (based on } \\
\text { symmetry-related } \\
\text { measurements) }\end{array}$ & None \\
\hline$T_{\min }$ & 0.114 & 0.679 & 0.804 & - \\
\hline$T_{\max }$ & 0.209 & 1.000 & 1.000 & - \\
\hline $\begin{array}{l}\text { No. of measured, } \\
\text { independent and } \\
\text { observed reflections }\end{array}$ & $5463,2660,1702$ & 13 298, 13 281, 12058 & $8292,4198,3144$ & $5827,1750,1297$ \\
\hline $\begin{array}{l}\text { Criterion for observed } \\
\text { reflections }\end{array}$ & $I>2 \sigma(I)$ & $I>2 \sigma(I)$ & $I>2 \sigma(I)$ & $I>2 \sigma(I)$ \\
\hline$R_{\text {int }}$ & 0.061 & 0.060 & 0.038 & 0.039 \\
\hline$\theta_{\max }\left({ }^{\circ}\right)$ & 27.5 & 27.7 & 28.9 & 27.5 \\
\hline Range of $h, k, l$ & $\begin{array}{l}-8 \Rightarrow h \Rightarrow 8 \\
-9 \Rightarrow k \Rightarrow 9 \\
-16 \Rightarrow l \Rightarrow 16\end{array}$ & $\begin{array}{l}-6 \Rightarrow h \Rightarrow 6 \\
-12 \Rightarrow k \Rightarrow 12 \\
-18 \Rightarrow l \Rightarrow 18\end{array}$ & $\begin{array}{l}-7 \Rightarrow h \Rightarrow 7 \\
-16 \Rightarrow k \Rightarrow 16 \\
-17 \Rightarrow l \Rightarrow 17\end{array}$ & $\begin{array}{l}-25 \Rightarrow h \Rightarrow 19 \\
-24 \Rightarrow k \Rightarrow 25 \\
-11 \Rightarrow l \Rightarrow 12\end{array}$ \\
\hline \multicolumn{5}{|l|}{ Refinement } \\
\hline Refinement on & $F^{2}$ & $F^{2}$ & $F^{2}$ & $F^{2}$ \\
\hline $\begin{array}{l}R\left[F^{2}>2 \sigma\left(F^{2}\right)\right] \\
\quad w R\left(F^{2}\right), S\end{array}$ & $0.038,0.091,0.87$ & $0.084,0.221,1.04$ & $0.042,0.097,0.98$ & $0.044,0.131,1.05$ \\
\hline No. of reflections & 2657 & 13281 & 4198 & 1750 \\
\hline No. of parameters & 118 & 182 & 190 & 100 \\
\hline H-atom treatment & $\begin{array}{l}\text { Constrained to parent } \\
\text { site }\end{array}$ & Riding model & $\begin{array}{l}\text { Constrained to parent } \\
\text { site }\end{array}$ & $\begin{array}{l}\text { Constrained to parent } \\
\text { site }\end{array}$ \\
\hline Weighting scheme & $\begin{array}{l}w=1 /\left[\sigma^{2}\left(F_{o}^{2}\right)+\right. \\
\left.\quad(0.048 P)^{2} P\right], \text { where } \\
\quad P=\left(F_{o}^{2}+2 F_{c}^{2}\right) / 3\end{array}$ & $\begin{array}{l}w=1 /\left[\sigma^{2}\left(F_{o}^{2}\right)+\right. \\
\left.\quad(0.098 P)^{2}+8.436 P\right] \\
\text { where } P=\left(F_{o}^{2}+\right. \\
\left.2 F_{c}^{2}\right) / 3\end{array}$ & $\begin{array}{l}w=1 /\left[\sigma^{2}\left(F_{o}^{2}\right)+\right. \\
\left.(0.0505 P)^{2}\right], \text { where } \\
P=\left(F_{o}^{2}+2 F_{c}^{2}\right) / 3\end{array}$ & $\begin{array}{c}w=1 /\left[\sigma^{2}\left(F_{o}^{2}\right)+\right. \\
\left.(0.0725 P)^{2}\right], \text { where } \\
P=\left(F_{o}^{2}+2 F_{c}^{2}\right) / 3\end{array}$ \\
\hline$(\Delta / \sigma)_{\max }$ & 0.001 & 0.001 & 0.001 & 0.001 \\
\hline$\Delta \rho_{\max }, \Delta \rho_{\min }\left(\mathrm{e} \AA^{-3}\right)$ & $1.26,-1.31$ & $3.35,-2.06$ & $0.59,-0.92$ & $0.45,-0.16$ \\
\hline
\end{tabular}

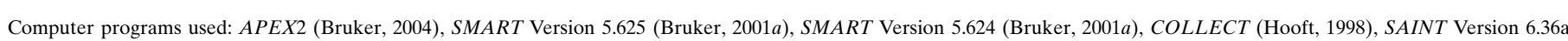

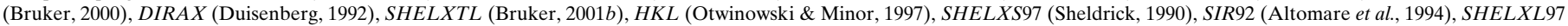
(Sheldrick, 1998), PLATON (Spek, 2003), enCIFer (Allen et al., 2004).

units of (1) and (11) each comprise single molecules, those of (2), (3), (4) and (4. $\left.\mathrm{CH}_{2} \mathrm{Cl}_{2}\right)$ comprise three, two, three and two molecules, respectively. The molecular structures of the alkylselenouracils and of 6- $n$-propyluracil are unremarkable, and the molecular structure of (4) is not affected by recrystallization as $\left(4 \cdot \mathrm{CH}_{2} \mathrm{Cl}_{2}\right)$ from dichloromethane. The $\mathrm{C}-\mathrm{Se}$ bond distances in (1)-(4) and in $\left(4 \cdot \mathrm{CH}_{2} \mathrm{Cl}_{2}\right)$ vary from 1.824 (2) to 1.848 (6) $\AA$ (Table 2). The two $\mathrm{C}=\mathrm{O}$ bond distances found in (11) are almost equal [1.2286(16), 1.2387 (16) $\AA$; Table 4] and are within the range of the $\mathrm{C}=\mathrm{O}$ bonds found for other similar compounds such as 1,3-di- methyl-6- $R$-trisubstituted uracils $[R=\mathrm{Me}, 1.233$ (3) $\AA$; $R=\mathrm{Et}$,

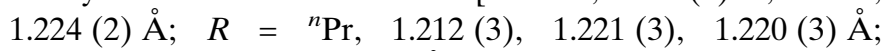
$R={ }^{n} \mathrm{Bu}, 1.220$ (5), 1.226 (5) А; Suwinska, 1995] and triethylammonium 2,4-dioxo-6-(1,1,2,2,3,3-hexafluoropropyl)-5-benzylsulfonyl-3H-2,4-dihydropyrimidine [1.235 (3), 1.237 (2) $\AA$ ] (Timoshenko et al., 2002).

\subsection{Extended structures of (1)-(4), $\left(4 \cdot \mathrm{CH}_{2} \mathrm{Cl}_{2}\right)$ and (11)}

The extended structures formed by the alkylselenouracils are worthy of comment; they depend primarily on hydrogen- 
bonding interactions between the two $\mathrm{N}-\mathrm{H}$ donor sites and the oxygen and selenium acceptor sites of the heteroatomic ring [see Scheme (II)]. Pertinent distances and angles associated with the hydrogen-bonding interactions in (1)-(4), $\left(4 \cdot \mathrm{CH}_{2} \mathrm{Cl}_{2}\right)$ and (11) are included in Table 3.

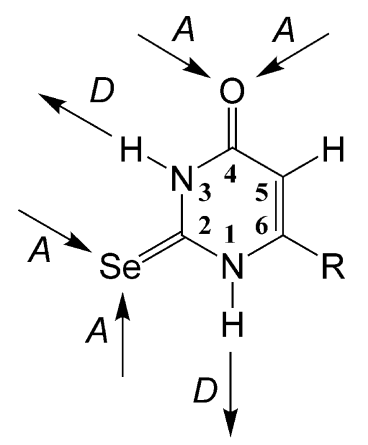

Scheme (II)

Analysis of the hydrogen-bonding parameters in these compounds confirms a range of intermolecular contacts with similar numbers of $\mathrm{N} 1-\mathrm{H} \cdots \mathrm{O}$ and $\mathrm{N} 3-\mathrm{H} \cdots \mathrm{O}$ and of $\mathrm{N} 1-$ $\mathrm{H}$. . Se and $\mathrm{N} 3-\mathrm{H} \cdots \mathrm{Se}$ interactions.

The extended structure of (1) is a two-dimensional sheet of topology $6^{3}$ (Fig. 7). A centrosymmetrically related pair of $\mathrm{N} 3-\mathrm{H} \cdots$ Se contacts form an $R_{2}^{2}(8)$ ring which links two molecules, thereby forming a dimeric unit. Each dimeric unit is bridged to four adjacent dimeric units through four single $\mathrm{N} 1-\mathrm{H} \cdots \mathrm{O}$ contacts to give a sheet of six-membered rings, each of which involves an $R_{6}^{6}(28)$ hydrogen-bonded motif (Table 3 ). The $6^{3}$ topology of the sheet, which is aligned
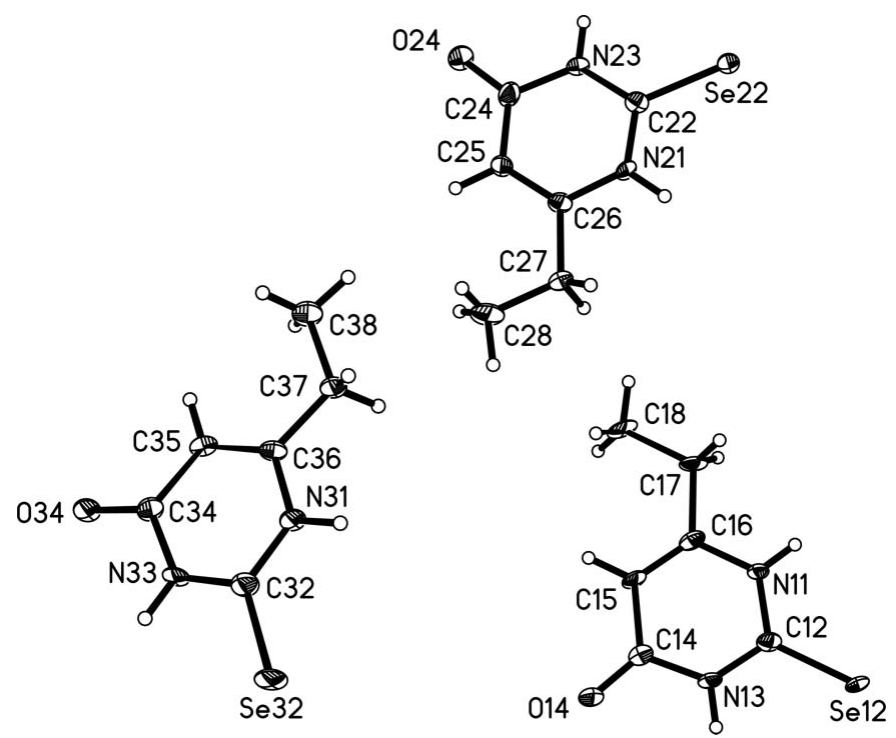

Figure 2

Displacement ellipsoid plot of (2) showing the atom-numbering scheme, with ellipsoids drawn at the $50 \%$ probability level. There are three independent molecules in the asymmetric unit.

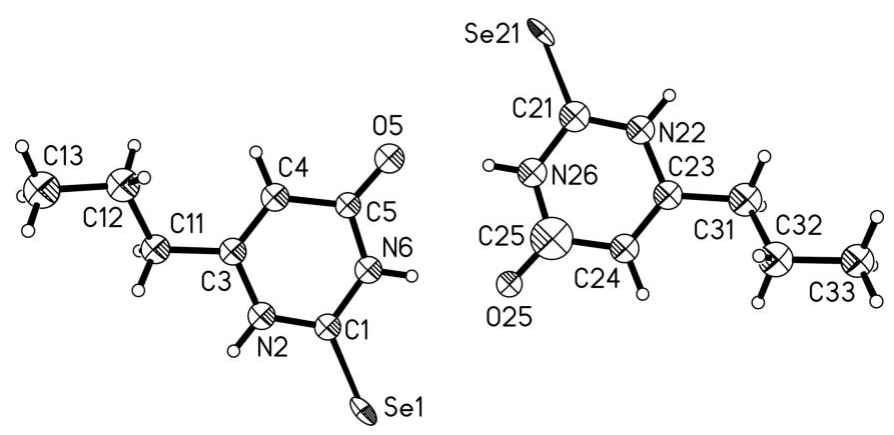

Figure 3

Displacement ellipsoid plot of (3) showing the atom-numbering scheme, with ellipsoids drawn at the $50 \%$ probability level. There are two independent molecules in the asymmetric unit.

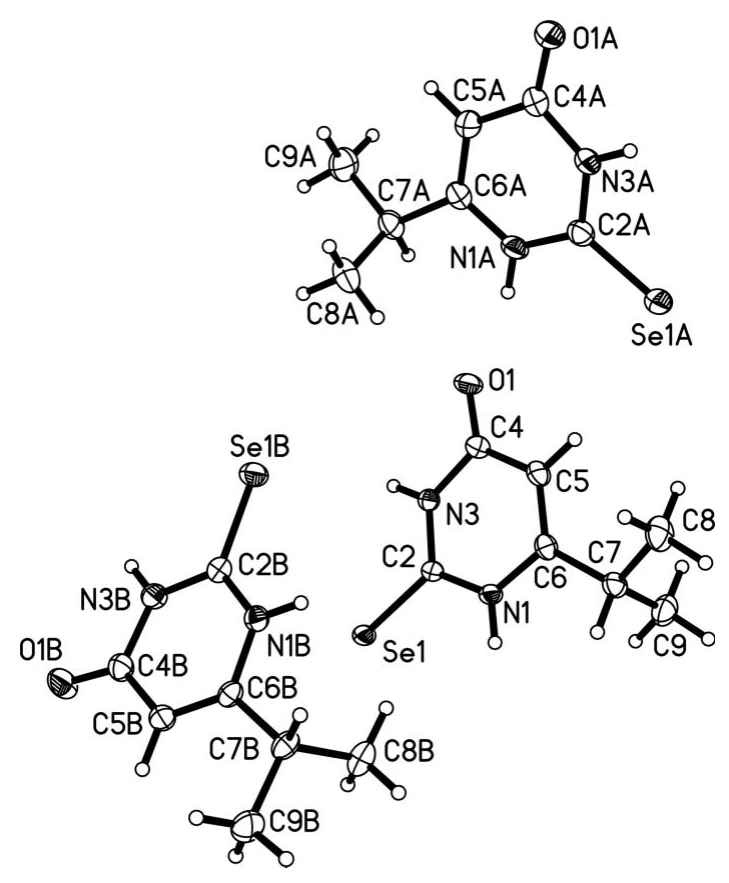

Figure 4

Displacement ellipsoid plot of (4) showing the atom-numbering scheme, with ellipsoids drawn at the $50 \%$ probability level. There are three independent molecules in the asymmetric unit.

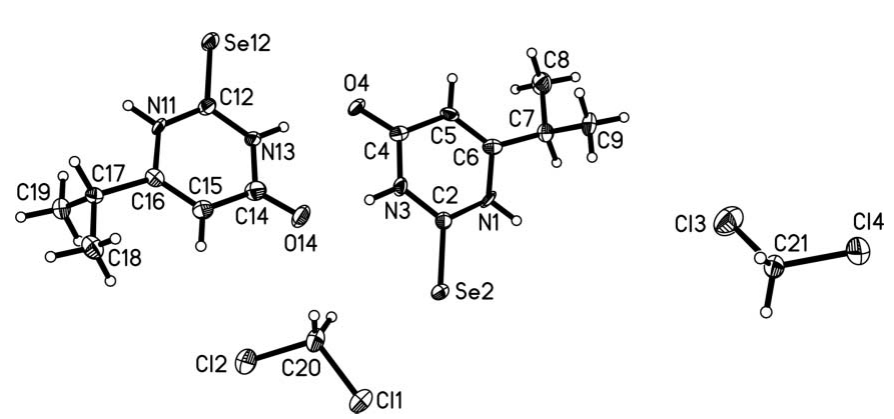

Figure 5

Displacement ellipsoid plot of $\left(4 \cdot \mathrm{CH}_{2} \mathrm{Cl}_{2}\right)$ showing the atom-numbering scheme, with ellipsoids drawn at the $50 \%$ probability level. Two independent molecules of (4) are solvated by two dichloromethane molecules in the asymmetric unit. 
Table 2

Molecular geometry parameters $\left(\AA{ }^{\circ},{ }^{\circ}\right)$ for (1) (2), (3), (4) and $\left(4 \cdot \mathrm{CH}_{2} \mathrm{Cl}_{2}\right)$.

\begin{tabular}{|c|c|c|c|c|c|c|c|c|c|}
\hline \multicolumn{2}{|l|}{$(1)$} & \multicolumn{2}{|l|}{ (2) } & \multicolumn{2}{|l|}{ (3) } & \multicolumn{2}{|l|}{ (4) } & \multicolumn{2}{|l|}{$\left(4 \cdot \mathrm{CH}_{2} \mathrm{Cl}_{2}\right)$} \\
\hline $\mathrm{Se} 2-\mathrm{C} 2$ & $1.832(2)$ & Se12-C12 & $1.835(6)$ & $\mathrm{Se} 1-\mathrm{C} 1$ & $1.837(16)$ & $\mathrm{Se} 1-\mathrm{C} 2$ & $1.843(2)$ & $\mathrm{Se} 2-\mathrm{C} 2$ & $1.829(6)$ \\
\hline $\mathrm{N} 1-\mathrm{C} 2$ & $1.352(3)$ & $\mathrm{Se} 22-\mathrm{C} 22$ & $1.848(6)$ & $\mathrm{Se} 21-\mathrm{C} 21$ & $1.835(16)$ & $\mathrm{Se} 1 A-\mathrm{C} 2 A$ & $1.824(2)$ & $\mathrm{Se} 12-\mathrm{C} 12$ & $1.825(5)$ \\
\hline $\mathrm{N} 3-\mathrm{C} 2$ & $1.353(3)$ & Se $32-C 32$ & $1.828(7)$ & $\mathrm{N} 2-\mathrm{C} 1$ & $1.32(2)$ & $\mathrm{Se} 1 B-\mathrm{C} 2 B$ & $1.835(2)$ & $\mathrm{N} 1-\mathrm{C} 2$ & $1.345(7)$ \\
\hline \multirow[t]{2}{*}{$\mathrm{O} 4-\mathrm{C} 4$} & $1.233(3)$ & $\mathrm{N} 11-\mathrm{C} 12$ & $1.354(8)$ & $\mathrm{N} 2-\mathrm{C} 3$ & $1.40(2)$ & $\mathrm{N} 1-\mathrm{C} 2$ & $1.348(3)$ & $\mathrm{N} 3-\mathrm{C} 2$ & $1.351(7)$ \\
\hline & & $\mathrm{N} 21-\mathrm{C} 22$ & $1.341(8)$ & $\mathrm{N} 22-\mathrm{C} 2$ & $1.39(2)$ & $\mathrm{N} 1 A-\mathrm{C} 2 A$ & $1.354(3)$ & $\mathrm{N} 11-\mathrm{C} 12$ & $1.355(7)$ \\
\hline $\mathrm{Se} 2-\mathrm{C} 2-\mathrm{N} 3$ & $121.99(16)$ & $\mathrm{N} 31-\mathrm{C} 32$ & $1.352(9)$ & $\mathrm{N} 6-\mathrm{C} 1$ & $1.39(2)$ & $\mathrm{N} 1 B-\mathrm{C} 2 B$ & $1.342(3)$ & $\mathrm{N} 13-\mathrm{C} 12$ & $1.349(7)$ \\
\hline \multirow[t]{19}{*}{$\mathrm{Se} 2-\mathrm{C} 2-\mathrm{N} 1$} & $121.90(15)$ & $\mathrm{N} 13-\mathrm{C} 12$ & $1.352(7)$ & $\mathrm{N} 26-\mathrm{C} 21$ & $1.39(2)$ & $\mathrm{N} 1-\mathrm{C} 6$ & $1.389(3)$ & $\mathrm{O} 4-\mathrm{C} 4$ & $1.232(7)$ \\
\hline & & $\mathrm{N} 23-\mathrm{C} 22$ & $1.349(7)$ & $\mathrm{O} 5-\mathrm{C} 5$ & $1.23(2)$ & $\mathrm{N} 1 A-\mathrm{C} 6 A$ & $1.383(3)$ & $\mathrm{O} 14-\mathrm{C} 14$ & $1.233(7)$ \\
\hline & & $\mathrm{N} 33-\mathrm{C} 32$ & $1.349(8)$ & $\mathrm{O} 25-\mathrm{C} 25$ & $1.24(2)$ & $\mathrm{N} 1 B-\mathrm{C} 6 B$ & $1.389(3)$ & & \\
\hline & & $\mathrm{O} 14-\mathrm{C} 14$ & $1.240(8)$ & $\mathrm{Se} 1-\mathrm{C} 1-\mathrm{N} 2$ & $123.3(13)$ & $\mathrm{C} 2-\mathrm{N} 3$ & $1.341(3)$ & $\mathrm{Se} 2-\mathrm{C} 2-\mathrm{N} 1$ & $123.0(4)$ \\
\hline & & $\mathrm{O} 24-\mathrm{C} 24$ & $1.242(8)$ & $\mathrm{Se} 1-\mathrm{C} 1-\mathrm{N} 6$ & $121.7(12)$ & $\mathrm{C} 2 A-\mathrm{N} 3 A$ & $1.351(3)$ & $\mathrm{Se} 2-\mathrm{C} 2-\mathrm{N} 3$ & $121.5(4)$ \\
\hline & & $\mathrm{O} 34-\mathrm{C} 34$ & $1.227(7)$ & $\mathrm{Se} 21-\mathrm{C} 21-\mathrm{N} 22$ & $123.9(13)$ & $\mathrm{C} 2 B-\mathrm{N} 3 B$ & $1.353(3)$ & $\mathrm{Se} 12-\mathrm{C} 12-\mathrm{N} 11$ & $123.5(4)$ \\
\hline & & & & $\mathrm{Se} 21-\mathrm{C} 21-\mathrm{N} 26$ & $121.2(12)$ & $\mathrm{N} 3-\mathrm{C} 4$ & $1.400(3)$ & $\mathrm{Se} 12-\mathrm{C} 12-\mathrm{N} 13$ & $121.1(4)$ \\
\hline & & $\mathrm{Se} 12-\mathrm{C} 12-\mathrm{N} 11$ & $123.2(4)$ & & & $\mathrm{N} 3 A-\mathrm{C} 4 A$ & $1.391(3)$ & & \\
\hline & & $\mathrm{Se} 22-\mathrm{C} 22-\mathrm{N} 21$ & $121.0(4)$ & & & $\mathrm{N} 3 B-\mathrm{C} 4 B$ & $1.395(3)$ & & \\
\hline & & $\mathrm{Se} 32-\mathrm{C} 32-\mathrm{N} 31$ & $123.7(5)$ & & & $\mathrm{O} 1-\mathrm{C} 4$ & $1.230(3)$ & & \\
\hline & & $\mathrm{Se} 12-\mathrm{C} 12-\mathrm{N} 13$ & $121.6(4)$ & & & $\mathrm{O} 1 A-\mathrm{C} 4 A$ & $1.237(3)$ & & \\
\hline & & $\mathrm{Se} 22-\mathrm{C} 22-\mathrm{N} 23$ & $122.8(4)$ & & & $\mathrm{O} 1 B-\mathrm{C} 4 B$ & $1.226(3)$ & & \\
\hline & & $\mathrm{Se} 32-\mathrm{C} 32-\mathrm{N} 33$ & $121.3(5)$ & & & & & & \\
\hline & & & & & & $\mathrm{Se} 1-\mathrm{C} 2-\mathrm{N} 1$ & $120.70(17)$ & & \\
\hline & & & & & & $\mathrm{Se} 1 A-\mathrm{C} 2 A-\mathrm{N} 1 A$ & $123.65(18)$ & & \\
\hline & & & & & & $\mathrm{Se} 1 B-\mathrm{C} 2 B-\mathrm{N} 1 B$ & $122.91(17)$ & & \\
\hline & & & & & & $\mathrm{Se} 1-\mathrm{C} 2-\mathrm{N} 3$ & $122.59(17)$ & & \\
\hline & & & & & & $\mathrm{Se} 1 A-\mathrm{C} 2 A-\mathrm{N} 3 A$ & $121.33(18)$ & & \\
\hline & & & & & & $\mathrm{Se} 1 B-\mathrm{C} 2 B-\mathrm{N} 3 B$ & $121.19(17)$ & & \\
\hline
\end{tabular}

parallel to the $(10 \overline{2})$ plane, results in a brick-wall architecture (Fig. 7).

The extended structures of (2) and (4) are analogous. They crystallize in the same space group with similar cell dimensions, the only difference being the alkyl group. Their extended structure (Fig. 8) is based on a chain of eightmembered rings which is aligned in the [101] direction in the $(21 \overline{2})$ plane. The three molecules in the asymmetric unit are involved in different hydrogen-bonding motifs; the first (numbered $\mathrm{C} 11$ etc.) acts as a two-donor one-acceptor $(2 \mathrm{D}-$ $1 A)$ species; the second (numbered $\mathrm{C} 21$ etc.) acts as a twodonor three-acceptor species $(2 D-3 A)$; the third (numbered $\mathrm{C} 31 \mathrm{etc}$.) acts as a two-donor two-acceptor species $(2 D-2 A)$. Each eight-membered ring is centrosymmetric and comprises two $2 D-1 A$ molecules, two $2 D-2 A$ molecules and four $2 D-3 A$ molecules. Pairs of centrosymmetrically related $2 D-3 A$ molecules are common to two adjacent rings, thereby giving

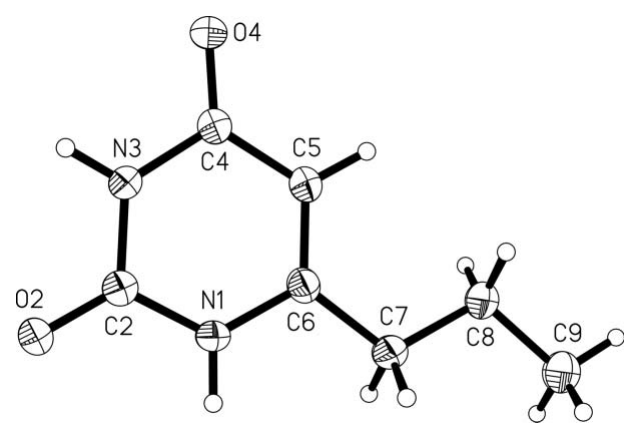

Figure 6

Displacement ellipsoid plot of (11) showing the atomic-numbering scheme, with ellipsoids drawn at the $50 \%$ probability level. the correct stoichiometry. The hydrogen-bonding interactions leading to the eight-membered ring comprise an $R_{8}^{6}(32)$ motif (Table 3). The aliphatic residues of the two $2 D-1 A$ and two $2 D-2 A$ molecules are located on the edges of the chain, preventing inter-chain hydrogen-bond formation (Fig. 8).

In (3), $R_{2}^{2}(8)$ hydrogen-bonding motifs link the two molecules of the asymmetric unit, which alternate along the $b$ axis to form a one-dimensional chain (Fig. 9). The $R_{2}^{2}(8)$ motifs differ in that one has oxygen acceptors and the other has selenium acceptors. The 1:1 adduct $\left(4 \cdot \mathrm{CH}_{2} \mathrm{Cl}_{2}\right)$ has a similar
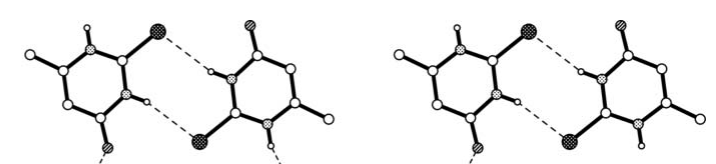

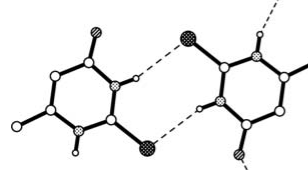<smiles>CCC1CC(O)C(C)C(C)C1C</smiles>

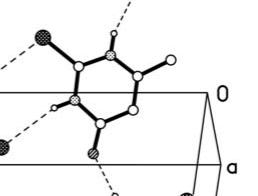
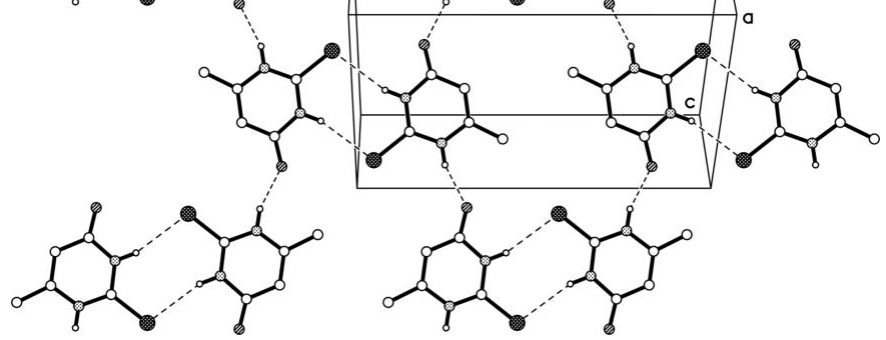

Figure 7

View of part of a sheet in the crystal structure of (1) showing the intermolecular $\mathrm{N}-\mathrm{H} \cdots \mathrm{O}$ and pairwise $\mathrm{N}-\mathrm{H} \cdots \mathrm{Se}$ interactions. Atoms are identified as follows: $\mathrm{Se}$, large cross-hatched circles; $\mathrm{O}$, hatched circles; $\mathrm{N}$, dotted circles; $\mathrm{C}$, intermediate open circles; $\mathrm{H}$, small open circles. 
Table 3

Hydrogen-bonding parameters $\left(\AA,^{\circ}\right)$ for (1)-(4), $\left(4 \cdot \mathrm{CH}_{2} \mathrm{Cl}_{2}\right)$ and (11) $[\mathrm{N}-\mathrm{H}=0.88 \AA ; \mathrm{C}-\mathrm{H}=0.95 \AA ; \lambda=0.6775 \AA$ for (1), (3) and (10)].

\begin{tabular}{|c|c|c|c|}
\hline Interaction & $\mathrm{H} \cdots Y(\AA)$ & $X \cdots Y(\AA)$ & $X-\mathrm{H} \cdots Y\left(^{\circ}\right)$ \\
\hline \multicolumn{4}{|l|}{ (1) } \\
\hline $\mathrm{N} 1-\mathrm{H} 1 A \cdots \mathrm{O} 4$ & 1.91 & $2.761(2)$ & 162 \\
\hline $\mathrm{N} 3-\mathrm{H} 3 A \cdots \mathrm{Se} 2$ & 2.63 & $3.4825(14)$ & 162 \\
\hline \multicolumn{4}{|l|}{ (2) } \\
\hline $\mathrm{N} 11-\mathrm{H} 11 \cdots \mathrm{O} 34$ & 1.93 & $2.805(6)$ & 171 \\
\hline $\mathrm{N} 23-\mathrm{H} 23 \cdots \mathrm{O} 14$ & 1.99 & $2.868(7)$ & 172 \\
\hline $\mathrm{N} 31-\mathrm{H} 31 \cdots \mathrm{O} 24$ & 1.97 & $2.829(7)$ & 166 \\
\hline $\mathrm{N} 13-\mathrm{H} 13 \cdots \mathrm{Se} 22$ & 2.47 & $3.340(5)$ & 169 \\
\hline $\mathrm{N} 21-\mathrm{H} 21 \cdots \mathrm{Se} 22$ & 2.58 & $3.442(5)$ & 167 \\
\hline $\mathrm{N} 33-\mathrm{H} 33 \cdots \mathrm{Se} 12$ & 2.55 & $3.417(6)$ & 170 \\
\hline \multicolumn{4}{|l|}{ (3) } \\
\hline N6-H6N ‥O25 & 1.96 & $2.80(2)$ & 161 \\
\hline $\mathrm{N} 26-\mathrm{H} 26 \mathrm{~N} \cdots \mathrm{O} 5$ & 1.99 & $2.84(2)$ & 162 \\
\hline N2-H2N $\cdots$ Se21 & 2.60 & $3.44(2)$ & 160 \\
\hline $\mathrm{N} 22-\mathrm{H} 22 \mathrm{~N} \cdots \mathrm{Se} 1$ & 2.59 & $3.46(2)$ & 167 \\
\hline \multicolumn{4}{|l|}{ (4) } \\
\hline $\mathrm{N} 1 A-\mathrm{H} 1 A A \cdots \mathrm{O} 1$ & 2.00 & $2.860(3)$ & 167 \\
\hline $\mathrm{N} 1 B-\mathrm{H} 1 B A \cdots \mathrm{O} 1 A$ & 1.92 & $2.797(3)$ & 177 \\
\hline $\mathrm{N} 3-\mathrm{H} 3 A \cdots \mathrm{O} 1 B$ & 1.91 & $2.782(3)$ & 169 \\
\hline $\mathrm{N} 1-\mathrm{H} 1 A \cdots \mathrm{Se} 1$ & 2.60 & $3.460(2)$ & 165 \\
\hline $\mathrm{N} 3 A-\mathrm{H} 3 A A \cdots \mathrm{Se} 1 B$ & 2.60 & $3.430(3)$ & 157 \\
\hline $\mathrm{N} 3 B-\mathrm{H} 3 B A \cdots \mathrm{Se} 1$ & 2.56 & $3.428(2)$ & 167 \\
\hline \multicolumn{4}{|l|}{$\left(4 \cdot \mathrm{CH}_{2} \mathrm{Cl}_{2}\right)$} \\
\hline $\mathrm{N} 3-\mathrm{H} 3 \cdots \mathrm{O} 14$ & 1.97 & $2.831(6)$ & 167 \\
\hline $\mathrm{N} 13-\mathrm{H} 13 \cdots \mathrm{O} 4$ & 1.98 & $2.854(6)$ & 172 \\
\hline $\mathrm{N} 1-\mathrm{H} 1 \cdots \mathrm{Se} 12$ & 2.55 & $3.424(5)$ & 175 \\
\hline 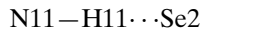 & 2.57 & $3.448(5)$ & 176 \\
\hline $\mathrm{C} 21-\mathrm{H} 21 A \cdots \mathrm{O} 4$ & 2.34 & $3.230(7)$ & 150 \\
\hline \multicolumn{4}{|l|}{ (11) } \\
\hline $\mathrm{N} 1-\mathrm{H} 1 A \cdots \mathrm{O} 4$ & 1.91 & $2.771(2)$ & 166 \\
\hline $\mathrm{N} 3-\mathrm{H} 3 A \cdots \mathrm{O} 2$ & 2.01 & $2.856(2)$ & 161 \\
\hline $\mathrm{C} 9-\mathrm{H} 9 A \cdots \mathrm{O} 4$ & 2.52 & $3.491(2)$ & 169 \\
\hline
\end{tabular}

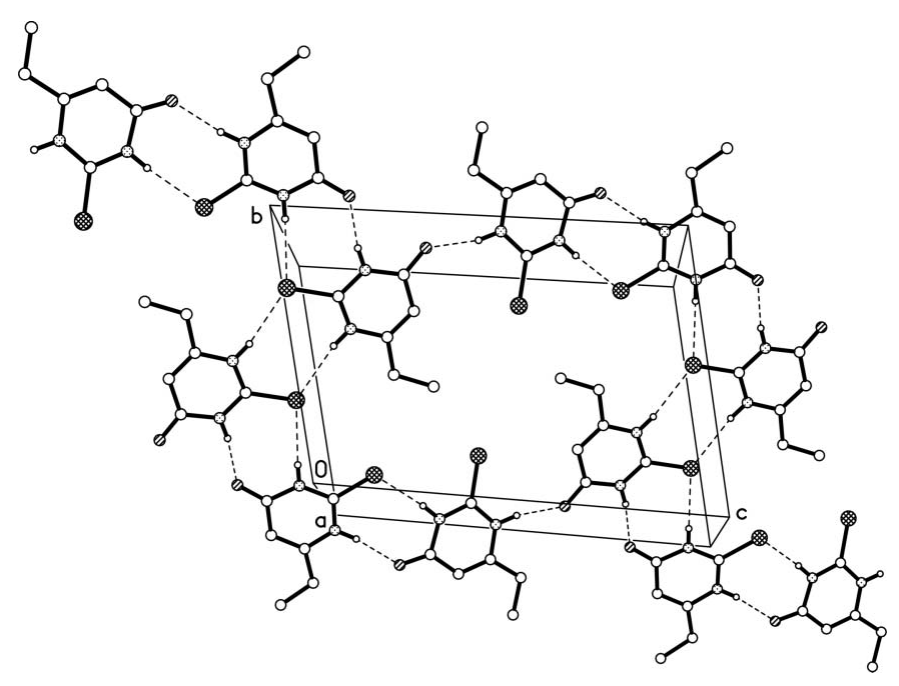

Figure 8

View of part of a sheet in the crystal structure of (2) showing the intermolecular $\mathrm{N}-\mathrm{H} \cdots \mathrm{O}$ and $\mathrm{N}-\mathrm{H} \cdots \mathrm{Se}$ interactions. Atoms are identified as in Fig. 7. extended structure (Fig. 10) to that of (3). In this case, however, the chain is aligned along the $b$ axis. One of the dichloromethane molecules is locked in position by a relatively short $\mathrm{C}-\mathrm{H} \cdots \mathrm{O}$ contact (Table 3 ).

It is interesting to note that a different extended structure is adopted by $6-n$-propyluracil (11), the uracil corresponding to (3). The asymmetric unit of (11) comprises a single molecule (Fig. 6), six of which combine to form a six-membered ring (Fig. 11). Linking each pair of molecules is an $R_{2}^{2}(8)$ hydrogenbonding motif, which forms part of the $R_{6}^{6}(24)$ hydrogenbonding motif (Table 3), generating the inner diameter of the six-membered ring. The rings assemble on a sheet parallel to the (001) plane. The only inter-ring interactions are a centrosymmetric pair of relatively long $\mathrm{C}-\mathrm{H} \cdots \mathrm{O}$ contacts between the pendant $\mathrm{CH}_{3}$ groups and carbonyl $\mathrm{O}$ atoms (Table 3). Six-membered ring formation is possible owing to the fact that the $R_{2}^{2}(8)$ motifs in (11) [see Scheme (III $\left.a\right)$ ], which comprise two $\mathrm{N}-\mathrm{H} \cdots \mathrm{O}$ contacts, are symmetrical and generate an internal ring angle of $120^{\circ}$. If (3) were to adopt a

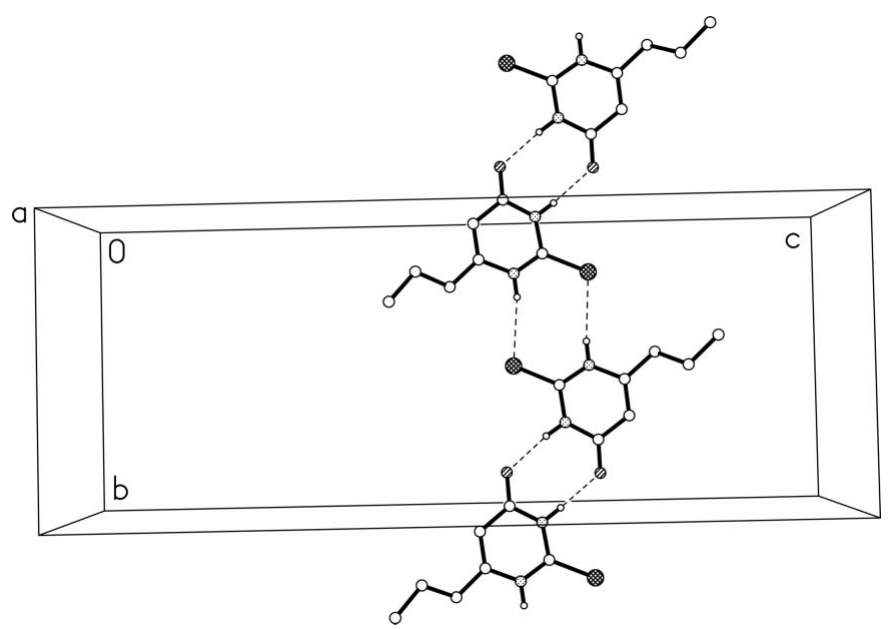

Figure 9

View of part of a chain in the crystal structure of (3) showing the intermolecular $\mathrm{N}-\mathrm{H} \cdots \mathrm{O}$ and $\mathrm{N}-\mathrm{H} \cdots \mathrm{Se}$ interactions. Atoms are identified as in Fig. 7.

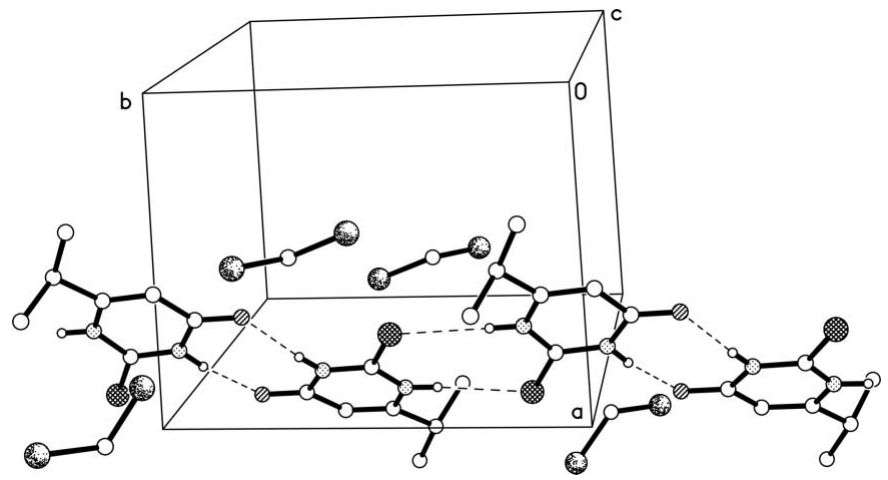

Figure 10

View of part of a chain in the crystal structure of $\left(4 \cdot \mathrm{CH}_{2} \mathrm{Cl}_{2}\right)$ showing the intermolecular $\mathrm{N}-\mathrm{H} \cdots \mathrm{O}$ and $\mathrm{N}-\mathrm{H} \cdots \mathrm{Se}$ interactions. Atoms are identified as in Fig. 7. 
Table 4

Molecular geometry parameters $\left(\AA,^{\circ}\right)$ for $(7),\left(9 \cdot 2 \mathrm{H}_{2} \mathrm{O}\right),(10)$ and (11).

\begin{tabular}{|c|c|c|c|c|c|c|c|}
\hline \multicolumn{2}{|l|}{ (7) } & \multicolumn{2}{|l|}{$\left(9 \cdot 2 \mathrm{H}_{2} \mathrm{O}\right)$} & \multicolumn{2}{|l|}{ (10) } & \multicolumn{2}{|l|}{ (11) } \\
\hline $\mathrm{I} 1-\mathrm{I} 2$ & $2.8928(10)$ & $\mathrm{Se} 2-\mathrm{Se} 2$ & $2.4328(9)$ & $\mathrm{Se} 2-\mathrm{Se} 2$ & $2.4427(6)$ & $\mathrm{O} 2-\mathrm{C} 2$ & $1.2286(16)$ \\
\hline $\mathrm{I} 1-\mathrm{Se}$ & $2.7807(11)$ & $\mathrm{Se} 2-\mathrm{C} 2$ & $1.925(4)$ & $\mathrm{Se} 2-\mathrm{C} 2$ & $1.922(4)$ & $\mathrm{O} 4-\mathrm{C} 4$ & $1.2387(16)$ \\
\hline $\mathrm{Se}-\mathrm{C} 2$ & $1.876(6)$ & $\mathrm{N} 1-\mathrm{C} 2$ & $1.283(6)$ & $\mathrm{N} 1-\mathrm{C} 2$ & $1.296(4)$ & $\mathrm{N} 1-\mathrm{C} 2$ & $1.3668(15)$ \\
\hline $\mathrm{N} 3-\mathrm{C} 2$ & $1.334(8)$ & $\mathrm{N} 3-\mathrm{C} 4$ & $1.431(6)$ & $\mathrm{N} 3-\mathrm{C} 4$ & $1.421(3)$ & $\mathrm{N} 3-\mathrm{C} 4$ & $1.3895(12)$ \\
\hline $\mathrm{N} 3-\mathrm{C} 4$ & $1.405(8)$ & $\mathrm{O} 4-\mathrm{C} 4$ & $1.233(5)$ & $\mathrm{O} 4-\mathrm{C} 4$ & $1.236(4)$ & & \\
\hline \multirow[t]{2}{*}{$\mathrm{O} 4-\mathrm{C} 4$} & $1.213(8)$ & $\mathrm{N} 3-\mathrm{C} 12$ & $1.420(5)$ & $\mathrm{N} 3-\mathrm{C} 12$ & $1.436(4)$ & $\mathrm{O} 2-\mathrm{C} 2-\mathrm{N} 3$ & $122.39(11)$ \\
\hline & & $\mathrm{N} 11-\mathrm{C} 12$ & $1.296(4)$ & $\mathrm{N} 11-\mathrm{C} 12$ & $1.271(4)$ & $\mathrm{O} 2-\mathrm{C} 2-\mathrm{N} 1$ & $122.45(11)$ \\
\hline \multirow{5}{*}{$\mathrm{I} 1-\mathrm{Se}-\mathrm{C} 2$} & & $\mathrm{O} 14-\mathrm{C} 14$ & $1.232(3)$ & $\mathrm{O} 14-\mathrm{C} 14$ & $1.230(4)$ & $\mathrm{N} 1-\mathrm{C} 2-\mathrm{N} 3$ & $115.15(8)$ \\
\hline & & $\mathrm{Se} 2-\mathrm{Se} 2-\mathrm{C} 2$ & 88.99 (14) & $\mathrm{Se} 2-\mathrm{Se} 2-\mathrm{C} 2$ & $89.44(8)$ & & \\
\hline & & $\mathrm{Se} 2-\mathrm{C} 2-\mathrm{N} 1$ & $114.7(3)$ & $\mathrm{Se} 2-\mathrm{C} 2-\mathrm{N} 1$ & $122.2(2)$ & & \\
\hline & & $\mathrm{Se} 2-\mathrm{C} 2-\mathrm{N} 3$ & $121.6(3)$ & $\mathrm{Se} 2-\mathrm{C} 2-\mathrm{N} 3$ & $115.1(2)$ & & \\
\hline & & $\mathrm{C} 2-\mathrm{Se} 2-\mathrm{Se} 2-\mathrm{C} 2$ & $-179.98(18)$ & $\mathrm{C} 2-\mathrm{Se} 2-\mathrm{Se} 2-\mathrm{C} 2$ & 180 & & \\
\hline
\end{tabular}

similar structure, the corresponding $R_{2}^{2}(8)$ motifs would be unsymmetrical as they would comprise one short $\mathrm{N}-\mathrm{H} \cdots \mathrm{O}$ contact and one long $\mathrm{N}-\mathrm{H}$...Se contact. Analysis of the structure of (2), which contains such assemblies, gives a value of $133^{\circ}$ for the internal ring angle [see Scheme (III $b$ )], which does not permit ring formation as it falls between the values required for seven- and eight-membered rings, namely 128.6 and $135^{\circ}$, respectively. Hence, (3) adopts a one-dimensional chain architecture with alternating $R_{2}^{2}(8)$ rings with pairs of oxygen and selenium acceptors (Fig. 9).

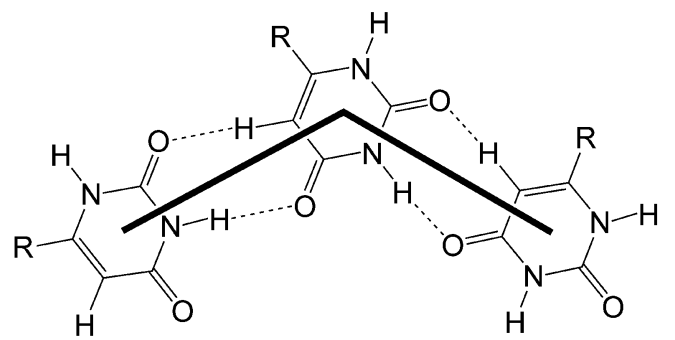

(a) Internal ring angle $120^{\circ}$ in (11)

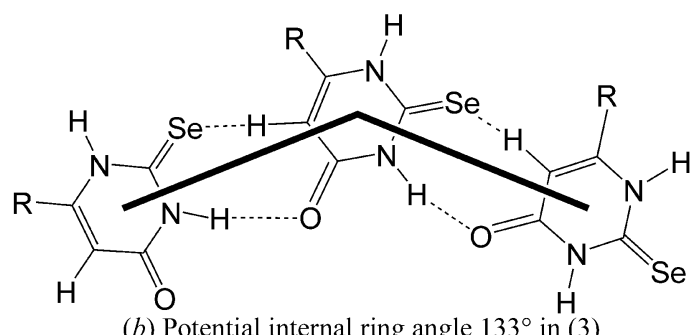

Scheme (III)

\subsection{Molecular structures of $(7),\left(9 \cdot 2 \mathrm{H}_{2} \mathrm{O}\right)$ and (10)}

Displacement ellipsoid plots of compounds (7), $\left(9 \cdot 2 \mathrm{H}_{2} \mathrm{O}\right)$ and (10) are shown in Figs. 12, 13 and 14, while their selected bond lengths and angles are listed in Table 4. Compound (7) exhibits the so-called 'spoke' structure typical of iodine adducts of selenium-containing compounds. The $\mathrm{I}-\mathrm{Se}-\mathrm{C}-\mathrm{N}$ torsion angles $\left[-0.5(6)^{\circ}\right.$ and $-179.3(6)^{\circ}$ for (7)], together with the $\mathrm{C}-\mathrm{Se}-\mathrm{I}$ and $\mathrm{Se}-\mathrm{I}-\mathrm{I}$ angles (Table 4), are consistent with a planar arrangement. This planarity, which is typical of charge-transfer complexes with a 'spoke' structure, is

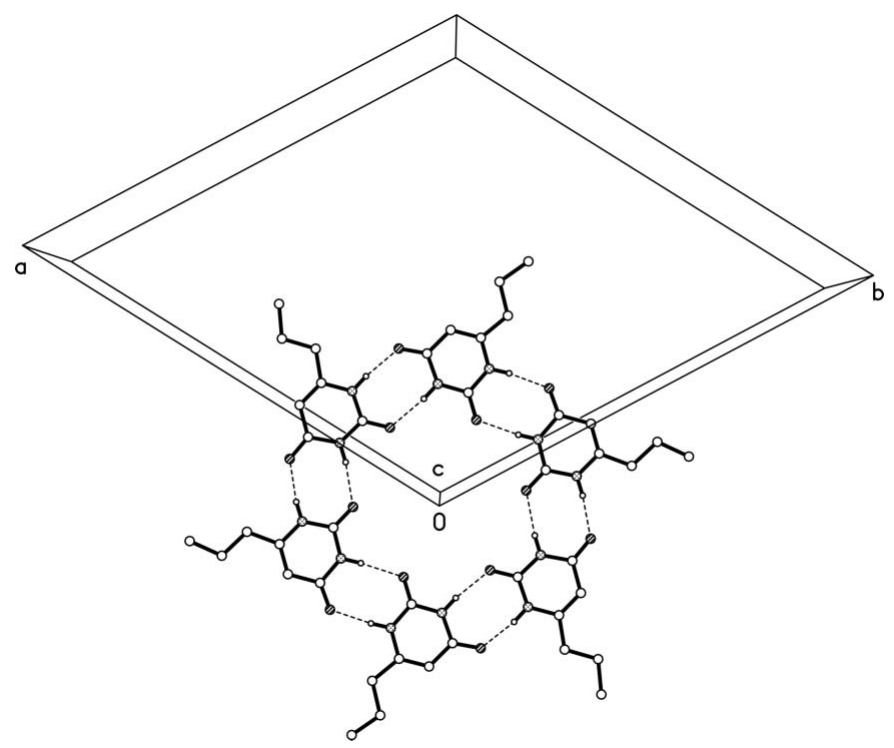

Figure 11

View of part of a sheet of (11) showing the intermolecular $\mathrm{N}-\mathrm{H} \cdots \mathrm{O}$ interactions. Atoms are identified as in Fig. 7.

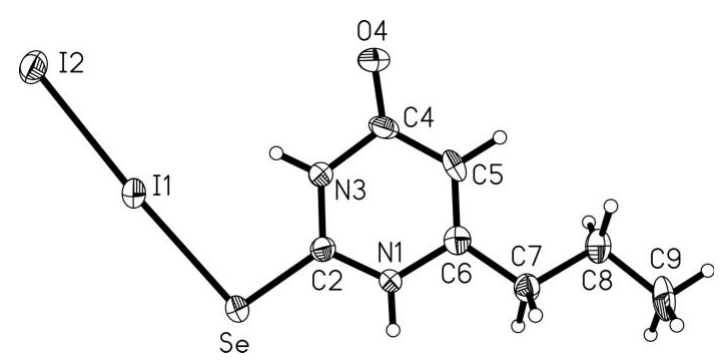

Figure 12

Displacement ellipsoid plot of (7) showing the atom-numbering scheme, with ellipsoids drawn at the $50 \%$ probability level. 
Table 5

Hydrogen-bonding parameters $\left(\AA{ }^{\circ},{ }^{\circ}\right)$ for $(7),\left(9 \cdot 2 \mathrm{H}_{2} \mathrm{O}\right)$ and $(10)(\mathrm{N}-\mathrm{H}=$ $0.88 \AA$; $\mathrm{C}-\mathrm{H}=0.95 \AA$ ).

\begin{tabular}{llll}
\hline Interaction & $\mathrm{H} \cdots Y(\AA)$ & $X \cdots Y(\AA)$ & $X-\mathrm{H} \cdots Y\left({ }^{\circ}\right)$ \\
\hline 7) & & & \\
$\mathrm{N} 1-\mathrm{H} 1 \mathrm{~N} \cdots \mathrm{O} 4$ & 1.85 & $2.698(8)$ & 161 \\
$\mathrm{~N} 3-\mathrm{H} 3 \mathrm{~N} \cdots \mathrm{I} 1$ & 2.66 & $3.371(5)$ & 139 \\
& & & \\
$\left(9 \cdot 2 \mathrm{H}_{2} \mathrm{O}\right)$ & & \\
$\mathrm{N} 13-\mathrm{H} 13 A \cdots \mathrm{O} 4$ & 1.88 & $2.560(5)$ & 133 \\
$\mathrm{~N} 13-\mathrm{H} 13 A \cdots \mathrm{O} 4{ }^{\prime}$ & 2.36 & $3.166(5)$ & 152 \\
$\mathrm{C} 5-\mathrm{H} 5 A \cdots \mathrm{O} 14$ & 2.48 & $3.304(5)$ & 145 \\
$\mathrm{O} 1 \cdots \mathrm{O} 14$ & & $2.861(5)$ & \\
$\mathrm{O} 1 \cdots \mathrm{O} 1^{\prime}$ & & $2.741(5)$ & \\
$\mathrm{O} 1 \cdots \mathrm{O} 1^{\prime}$ & & $2.770(5)$ & \\
$(10)$ & & & \\
$\mathrm{N} 13-\mathrm{H} 13 A \cdots \mathrm{O} 4$ & 1.84 & $2.534(3)$ & 134 \\
$\mathrm{C} 5-\mathrm{H} 5 A \cdots \mathrm{O} 4$ & 2.59 & $3.535(4)$ & 176 \\
$\mathrm{C} 15-\mathrm{H} 15 A \cdots \mathrm{O} 14$ & 2.61 & $3.562(4)$ & 175 \\
\hline
\end{tabular}

enhanced by an intramolecular $\mathrm{N}-\mathrm{H} \cdots \mathrm{I}$ hydrogen bond between N3 and I1 (Table 5; Fig. 15).

The I-I interatomic distance of 2.8928 (10) $\AA$ in (7) is longer than that in either the gas phase $(2.677 \AA$ : Pauling, 1960) or crystalline diiodine [2.715 (6) A at $110 \mathrm{~K}$ : van Bolhuis et al., 1967], presumably owing to the Se $\cdots I$ interaction. It is, however, the shortest such distance measured for a diiodineselenoamide complex (Antoniadis et al., 2006) suggesting minimal perturbation resulting from the long Se $\cdots$ I contact. Interestingly, correlation of the available Se $\cdots \mathrm{I}$ and $\mathrm{I} \cdots \mathrm{I}$ distance data (see Antoniadis et al., 2006) shows there to be a linear relationship between the two, which is quantified by the expression: $d(\mathrm{Se} \cdots \mathrm{I})=-0.7981 d(\mathrm{I} \cdots \mathrm{I})+5.0983, R^{2}=0.9805$. The I-I bond order of 0.547 calculated for (7) using the expression of Pauling (1960) is the highest such bond order for

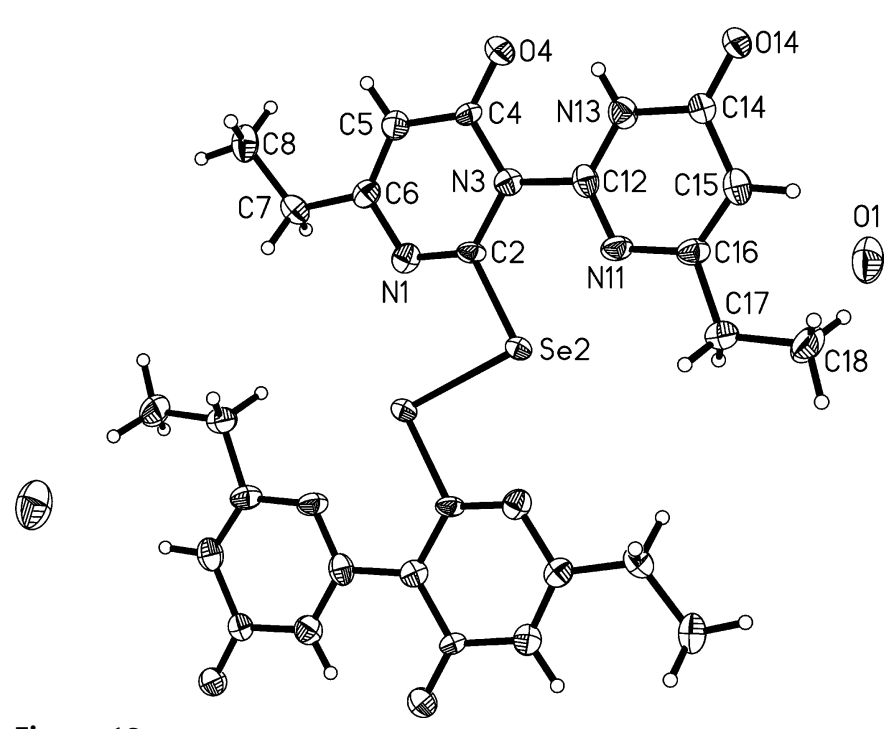

Figure 13

Displacement ellipsoid plot of molecule $\left(9 \cdot 2 \mathrm{H}_{2} \mathrm{O}\right)$ showing the atomicnumbering scheme, with ellipsoids drawn at the $50 \%$ probability level. The diselenide link is formed between $\mathrm{Se} 2$ and its symmetry equivalent at $(1-x,-y, 1-z)$. The water $\mathrm{H}$ atoms were not located. selenoamide-diiodine complexes. All these data are consistent with a very weak Se...I interaction. Bigoli et al. $(1996,1999)$ have separated iodine adducts of sulfur donors into three classes on the basis of I ...I bond order. Applying the same criteria to iodine adducts of selenium donors, (7) falls into the

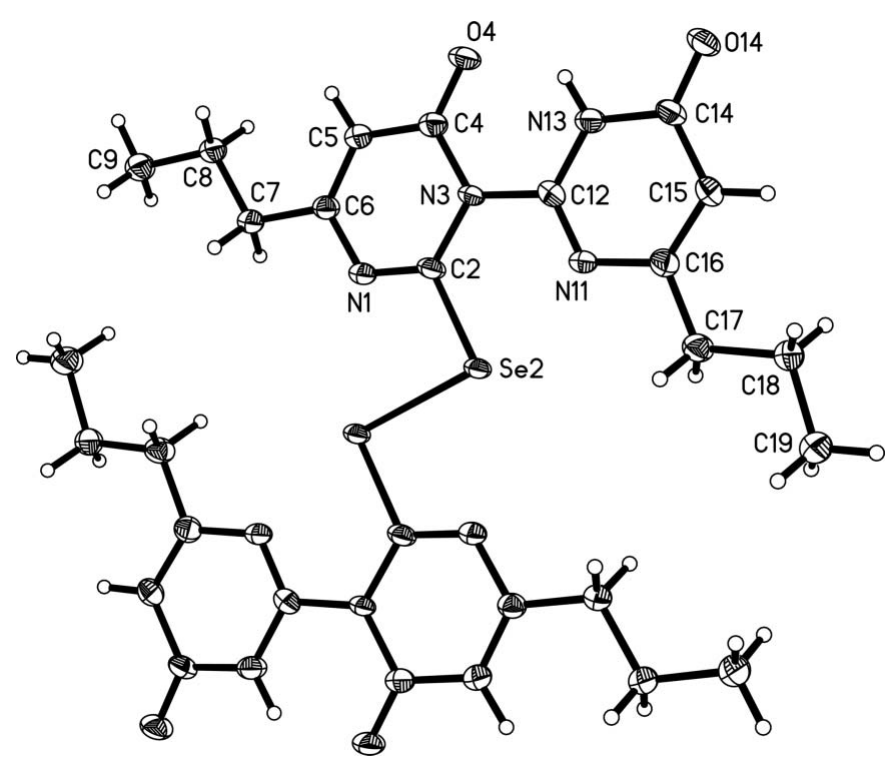

Figure 14

Displacement ellipsoid plot of molecule (10) showing the atomicnumbering scheme, with ellipsoids drawn at the $50 \%$ probability level. The diselenide link is formed between $\mathrm{Se} 2$ and its symmetry equivalent at $(-x,-y,-z)$.

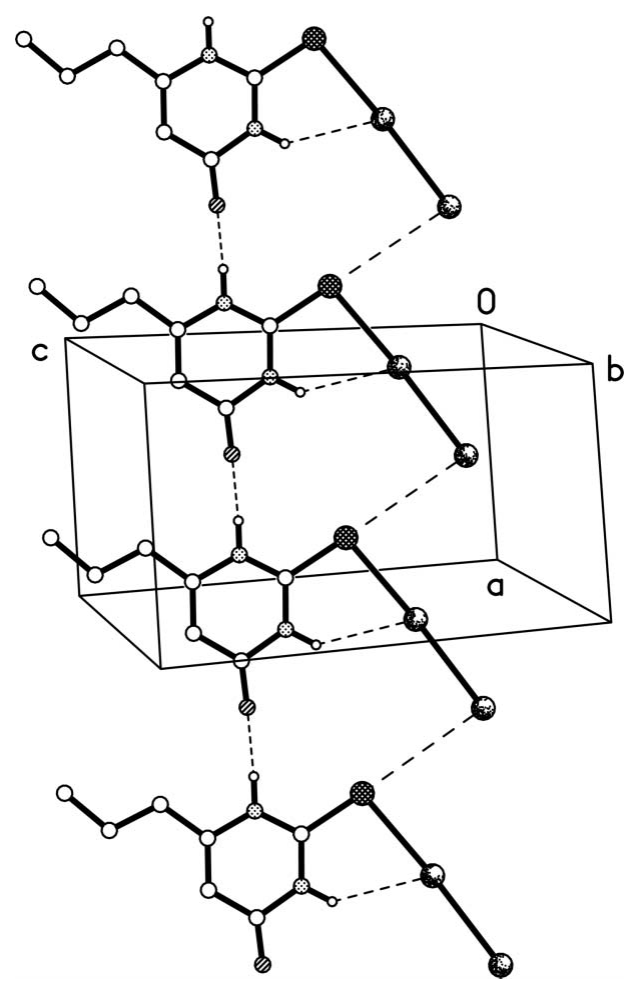

Figure 15

View of part of a chain in the crystal structure of (7) showing the intermolecular $\mathrm{N}-\mathrm{H} \cdots \mathrm{O}$ and $\mathrm{C}-\mathrm{H} \cdots \mathrm{I}$ and $\mathrm{Se} \cdots \mathrm{I}$ interactions. Atoms are identified as in Fig. 7. 
intermediate, rather than the weakest, classification which would require an I …I distance of $3.92 \AA$, which is close to double the van der Waals radius of iodine $(1.98 \AA)$. Therefore, a third type of $\mathrm{Se}-\mathrm{I}$ adduct seems very unlikely. Although the $\mathrm{C}-\mathrm{Se}$ distance in (7) [1.876 (6) $\AA]$ is slightly longer than that for the corresponding free selenouracil (3) [average 1.836 (11) $\AA$; Table 2], it is similar to the $\mathrm{C}-\mathrm{Se}$ distances found for the other charge-transfer complexes (see Antoniadis et al., 2006).

Re-crystallization of (6) and (7) from acetone solutions results in the formation of the diselenides $\left(9 \cdot 2 \mathrm{H}_{2} \mathrm{O}\right)$ and $(10)$, respectively [see Scheme (I)]. Their molecular structures consist of two centrosymmetrically related selenoamide ligands, which have been $N$-substituted by a deselenated ligand molecule, linked through an $\mathrm{Se}-\mathrm{Se}$ bond to form the diselenide (Figs. 13 and 14). The presence of a crystallographic inversion centre between the selenoamides fixes the $\mathrm{C}-\mathrm{Se}-$ $\mathrm{Se}-\mathrm{C}$ torsion angle at $180^{\circ}$, which is unusual for diselenoamides. The majority of those listed have torsion angles between 50 and $93^{\circ}$, the only other example of a $180^{\circ}$ angle

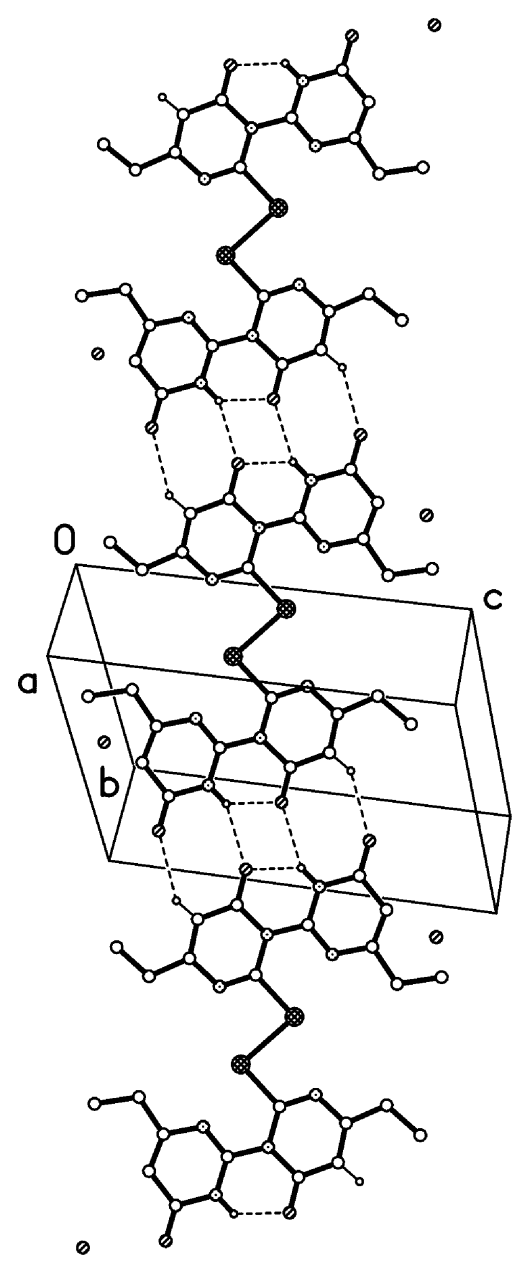

Figure 16

View of part of a sheet of $\left(9 \cdot 2 \mathrm{H}_{2} \mathrm{O}\right)$ showing the inter- and intramolecular $\mathrm{N}-\mathrm{H} \cdots \mathrm{O}$ and intermolecular $\mathrm{C}-\mathrm{H} \cdots \mathrm{O}$ interactions. Atoms are identified as in Fig. 7. being that in $N, N, N^{\prime}, N^{\prime}$-tetraethylthiuramdiselenide (Dietzsch et al., 1998).

The $\mathrm{Se}-\mathrm{Se}$ bond distances in $\left(9 \cdot 2 \mathrm{H}_{2} \mathrm{O}\right)[2.4328(9) \AA]$ and (10) $[2.4427$ (6) $\AA]$ fall within the range observed in other diselenoamides (2.34-2.59 $\AA$; Antoniadis et al., 2006). The C$\mathrm{Se}$ bond lengths in $\left(9 \cdot 2 \mathrm{H}_{2} \mathrm{O}\right)$ [1.925 (4) $\left.\AA\right]$ and (10) [1.922 (4) $\AA$ ], although longer than those of the corresponding free ligands (2) [mean C-Se 1.838 (5) $\AA$ ] and (3) [mean CSe 1.836 (11) $\AA$ ], fall in the range of other such compounds (1.874-1.952 ̊; Antoniadis et al., 2006). Molecules $\left(9 \cdot 2 \mathrm{H}_{2} \mathrm{O}\right)$ and (10) are neutral diselenoamides with two unequal $\mathrm{C}-\mathrm{N}$ bond distances, as expected for such compounds. These bond distances become equal in the case of ionic diselenoamides. The $\mathrm{Se}-\mathrm{Se}-\mathrm{C}$ bond angles in $\left(9 \cdot 2 \mathrm{H}_{2} \mathrm{O}\right)\left[88.99(14)^{\circ}\right]$ and $(10)$ $\left[89.44(8)^{\circ}\right]$ are at the lower end of the range found for diselenoamides.

\subsection{Extended structures of (7), $\left(9 \cdot 2 \mathrm{H}_{2} \mathrm{O}\right)$ and (10)}

The extended structure of (7) is based on a linear chain aligned along the $b$ axis (Fig. 15). The principal contact between adduct molecules is an intermolecular $\mathrm{N}-\mathrm{H} \cdots \mathrm{O}$ hydrogen bond (Table 5). It is supported by an interaction between the terminal iodine and the selenium atom [Se $\cdots \mathrm{I} 2$ $(x-1, y, z) 3.862(2) \AA$ A I $1-\mathrm{I} 2 \cdots \mathrm{Se}(1+x, y, z) 87.02(3)$; $\mathrm{C} 2-\mathrm{Se} \cdots \mathrm{I} 2(x-1, y, z) 171.3(2) ; \mathrm{I} 1-\mathrm{Se} \cdots \mathrm{I} 2(x-1, y, z)$ 83.82 (3); C2-Se...I1 $\left.96.9(2)^{\circ}\right]$.

The extended structures of $\left(9 \cdot 2 \mathrm{H}_{2} \mathrm{O}\right)$ and $(10)$ are similar. They form chains aligned along the [210] and [101] directions, respectively, as shown in Figs. 16 and 17, respectively. The principal links between molecules in $\left(9 \cdot 2 \mathrm{H}_{2} \mathrm{O}\right)$ are $\mathrm{N}-\mathrm{H} \cdots \mathrm{O}$ and in (10) are $\mathrm{C}-\mathrm{H} \cdots \mathrm{O}$ hydrogen bonds. The reason for the

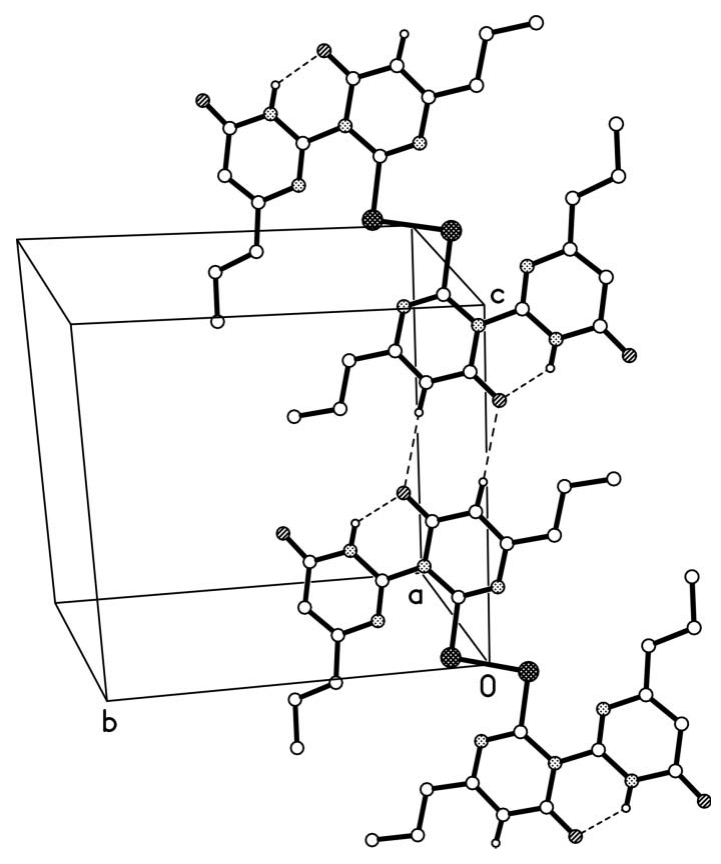

Figure 17

View of part of a sheet of (10) showing the intramolecular $\mathrm{N}-\mathrm{H} \cdots \mathrm{O}$ and intermolecular $\mathrm{C}-\mathrm{H} \cdots \mathrm{O}$ interactions. Atoms are identified as in Fig. 7. 
difference is not clear. In the case of $\left(9 \cdot 2 \mathrm{H}_{2} \mathrm{O}\right)$, the $\mathrm{N}-\mathrm{H} \cdots \mathrm{O}$ contact is quite long at 3.166 (5) $\AA$, and by inference weak, owing to bifurcation of the hydrogen bond, the other contact being an intramolecular interaction of 2.560 (5) $\AA$. The intermolecular contact is supported by a relatively short $\mathrm{C}-\mathrm{H} \cdots \mathrm{O}$

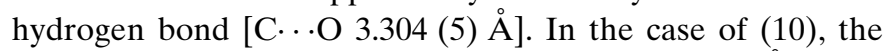
$\mathrm{N}-\mathrm{H} \cdots \mathrm{O}$ intramolecular contact [N..O 2.534 (3) $\AA$ ] is an independent interaction, the molecules being linked by a pair of centrosymmetrically related, somewhat long, $\mathrm{C}-\mathrm{H} \cdots \mathrm{O}$ hydrogen bonds [CO 3.535 (4) $\AA$ ] which form an $R_{2}^{2}(8)$ motif (Fig. 17).

In both structures the chains are stacked to give twodimensional sheet architectures parallel to $(1 \overline{2} 0)$ for $\left(9 \cdot 2 \mathrm{H}_{2} \mathrm{O}\right)$ and to (121) for (10). In the case of $\left(9 \cdot 2 \mathrm{H}_{2} \mathrm{O}\right)$ the chains are linked through hydrogen-bonded water molecules which not only form the sheet but also link the sheets into a threedimensional structure. Although it was not possible to locate the water $\mathrm{H}$ atoms, the proposed interactions are supported by the geometries around the $\mathrm{O}$ atoms. Thus, $\mathrm{O} \cdots \mathrm{O}$ distances range from 2.741 (5) through 2.770 (5) to 2.861 (5) $\AA$, while $\mathrm{O} \cdots \mathrm{O} \cdots \mathrm{O}$ angles range from 109.9 (2) through 122.5 (3) to $123.7(2)^{\circ}$. There are no aromatic stacking interactions owing to a staggered molecular arrangement. The only other possible interaction linking the sheets is an Se...Se contact, but the interatomic distance is extremely long [4.125 (2) $\mathrm{A}]$. In the case of (10) the chains are linked into two-dimensional sheets by relatively long $\mathrm{C}-\mathrm{H} \cdots \mathrm{O}$ contacts [C $\cdots \mathrm{O} 3.562$ (4) $\AA$; Table 5]. Hydrogen-bonded contacts between sheets are not feasible and the staggered molecular arrangement precludes aromatic stacking interactions. The only possible interaction is an Se...Se contact, but the interatomic distance of 3.961 (2) $\AA$ is rather long.

\section{Concluding remarks}

The architectures of the extended structures of the 6-alkyl-2selenouracils (1)-(4) and $\left(4 \cdot \mathrm{CH}_{2} \mathrm{Cl}_{2}\right)$ rely heavily on $R_{2}^{2}(8)$ hydrogen-bonded rings. All three types of rings, namely those based on two oxygen acceptors [O/O; see Scheme (IVa)], two selenium acceptors [Se/Se; see Scheme (IV $b$ )] and a mixture of one oxygen and one selenium acceptor $[\mathrm{O} / \mathrm{Se}$; see Scheme $(\mathrm{IV} c)]$, are found in the 6-alkyl-2-selenouracils. The most common is the $\mathrm{Se} / \mathrm{Se}$ ring; it appears in the extended structures of all five compounds [(1)-(4) and $\left.\left(4 \cdot \mathrm{CH}_{2} \mathrm{Cl}_{2}\right)\right]$. The other two types of ring appear twice only, the $\mathrm{O} / \mathrm{O}$ ring in (3) and $\left(4 \cdot \mathrm{CH}_{2} \mathrm{Cl}_{2}\right)$ and the $\mathrm{O} / \mathrm{Se}$ ring in (2) and (4). The simplest structures are those of (3) and $\left(4 \cdot \mathrm{CH}_{2} \mathrm{Cl}_{2}\right)$, where alternating $\mathrm{Se} / \mathrm{Se}$ and $\mathrm{O} / \mathrm{O}$ rings give rise to one-dimensional chain constructs (Figs. 9 and 10). In (1), Se/Se rings form dimers which are linked through $\mathrm{N}-\mathrm{H} \cdots \mathrm{O}$ hydrogen bonds to give a two-dimensional sheet of topology $6^{3}$ (Fig. 7). In (2) and (4), $\mathrm{Se} / \mathrm{Se}$ and $\mathrm{O} / \mathrm{Se}$ rings alternate to form chains of 6-alkyl-2selenouracils, which are linked through $\mathrm{N}-\mathrm{H} \cdots \mathrm{O}$ hydrogen bonds to give a two-dimensional sheet (Fig. 8). O/O $R_{2}^{2}(8)$ hydrogen-bonded rings are also found in the extended structure of 6- $n$-propyl-2-uracil (11), where they generate a six- membered paddle-wheel ring with pendant propyl groups (Fig. 11).

The extended structures of the diselenide oxidation products $\left(9 \cdot 2 \mathrm{H}_{2} \mathrm{O}\right.$ and 10$)$ also depend on $\mathrm{O} / \mathrm{O} R_{2}^{2}(8)$ hydrogen-bonded rings. However, in $\left(9 \cdot 2 \mathrm{H}_{2} \mathrm{O}\right)$ the donors are a mixture of $\mathrm{C}-\mathrm{H}$ and $\mathrm{N}-\mathrm{H}$ moieties [see $(\mathrm{IV} d)$ ] while in (10) they are exclusively $\mathrm{C}-\mathrm{H}$ donors. In both cases the molecules are linked to give rise to chain architectures (Figs. 16 and 17).

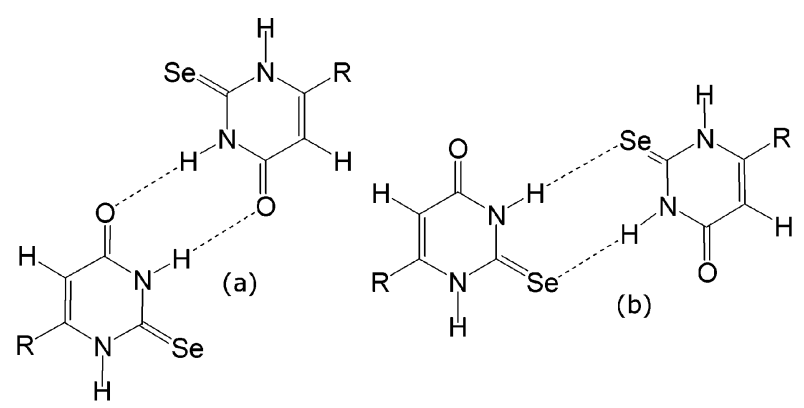

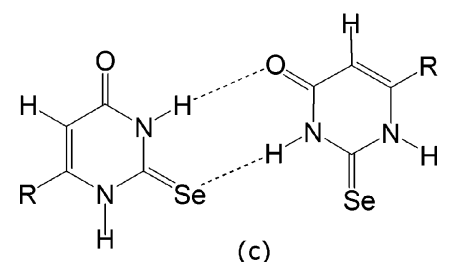

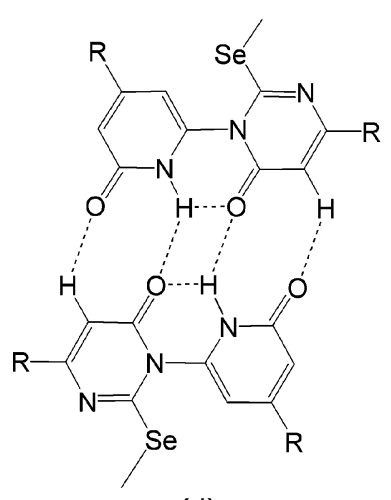

(d)

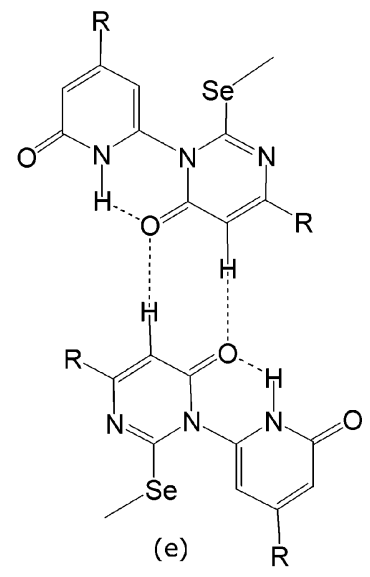

Scheme (IV) (e)
The $\mathrm{O}$ atoms of all five 6-alkyl-2-selenouracil and of the single 6- $n$-propyl-2-uracil structures act as acceptors to just one hydrogen-bonded contact. The Se atoms behave similarly except in (2) and (4). Of the Se atoms of the three molecules in the asymmetric unit of (2) and (4), one acts as a dual acceptor, one as a single acceptor while the third is not involved in any hydrogen bonds (Fig. 8). The situation in the oxidation products $\left(9 \cdot 2 \mathrm{H}_{2} \mathrm{O}\right.$ and 10$)$ is complicated by the existence of intramolecular $\mathrm{N}-\mathrm{H} \cdots \mathrm{O}$ hydrogen bonds. In $\left(9 \cdot 2 \mathrm{H}_{2} \mathrm{O}\right)$ one oxygen acts as a dual acceptor to intra- and intermolecular $\mathrm{N}-\mathrm{H} \cdots \mathrm{O}$ hydrogen bonds and a second oxygen acts as a single acceptor in an intermolecular $\mathrm{C}-\mathrm{H} \cdots \mathrm{O}$ hydrogen bond. All three contacts generate the one-dimensional chain structure (Fig. 16). In (10), one oxygen acts as a dual acceptor 
to an intramolecular $\mathrm{N}-\mathrm{H} \cdots \mathrm{O}$ and an intermolecular $\mathrm{C}-$ $\mathrm{H}$... O hydrogen bond to generate the one-dimensional chain structure, while a second oxygen acts as a single acceptor in an interchain $\mathrm{C}-\mathrm{H} \cdots \mathrm{O}$ hydrogen bond (Fig. 17).

We thank the Marie Curie Foundation for funding (to CDA) as part of the Marie Curie Host Fellowship HPMT-CT2001-00376 COSMIC, and the graduate program in Bioinorganic Chemistry based at Ioannina, Greece and coordinated by NH. We thank EPSRC (UK) for the award of diffractometers and for data collection by the EPSRC National Service for Crystallography at the University of Southampton, UK, and at the Daresbury Laboratory Synchrotron Radiation Source. MS acknowledges receipt of a Royal Society-Leverhulme Trust Senior Research Fellowship and of a Royal Society Wolfson Merit Award.

\section{References}

Allen, F. H., Johnson, O., Shields, G. P., Smith, B. R. \& Towler, M. (2004). J. Appl. Cryst. 37, 335-338.

Altomare, A., Burla, M. C., Camalli, M., Cascarano, G., Giacovazzo, C., Guagliardi, A. \& Polidori, G. (1994). J. Appl. Cryst. 27, 435.

Antoniadis, C. D., Hadjikakou, S. K., Hadjiliadis, N., Baril, M. \& Butler, I. S. (2006). Chem. Eur. J. DOI: 10.1002/chem.200501455.

Aragoni, M. C., Arca, A., Demartin, F., Devillanova, F. A., Garau, A., Isaia, F., Lippolis, V. \& Verani, G. (1999). Trends Inorg. Chem. 6, 118.

Aragoni, M. C., Arca, M., Blake, A. J., Devillanova, F. A., Du Mont, W.-W., Garau, A., Isaia, F., Lippolis, V., Verani, G. \& Wilson, C. (2001). Angew. Chem. Int. Ed. 40, 4229-4232.

Bent, H. A. (1968). Chem. Rev. 68, 587-648.

Bigoli, F., Deplano, P., Ienco, A., Mealli, C., Mercuri, M. L., Pellinghelli, M. A., Pintus, G., Saba, G. \& Trogu, E. F. (1999). Inorg. Chem. 38, 4626-4636.

Bigoli, F., Deplano, P., Mercuri, M. L., Pellinghelli, M. A., Sabatini, A., Trogu, E. F. \& Vacca, A. (1996). J. Chem. Soc. Dalton Trans. pp. 3583-3589.

Blake, A. J., Devillanova, F. A., Garau, A., Gilby, L. M., Gould, R. O., Isaia, F., Lippolis, V., Parsons, S., Radek, C. \& Schröder, M. (1998). J. Chem. Soc. Dalton Trans. pp. 2037-2046.
Blake, A. J., Devillanova, F. A., Gould, R. O., Li, W.-S., Lippolis, V., Parsons, S., Radek, C. \& Schröder, M. (1998). Chem. Soc. Rev. 27, 195-205.

Blake, A. J., Gould, R. O., Parsons, S., Radek, C. \& Schröder, M. (1995). Angew. Chem. Int. Ed. Engl. 34, 2374-2376.

Blake, A. J., Li, W.-S., Lippolis, V., Parsons, S., Radek, C. \& Schröder, M. (1998). Angew. Chem. Int. Ed. Engl. 37, 293-296.

Bjorvatten, T. (1963). Acta Chem. Scand. 17, 2292-2300.

Bolhuis, F. van, Koster, P. B. \& Migchelsen, T. (1967). Acta Cryst. 23, 90-91.

Bruker (2000). SAINT. Version 6.36a. Bruker AXS Inc., Madison, Wisconsin, USA.

Bruker (2001a). SMART. Version 5.624 or 5.625. Bruker AXS Inc., Madison, Wisconsin, USA.

Bruker (2001b). SHELXTL. Bruker AXS Inc., Madison, Wisconsin, USA.

Bruker (2004). APEX2. Version 1.0-27. Bruker AXS Inc., Madison, Wisconsin, USA.

Chao, G. Y. \& McCullough, J. D. (1961). Acta Cryst. 14, 940945.

Dahl, T. \& Hassel, O. (1965). Acta Chem. Scand. 19, 20002001.

Dasent, W. E. (1965). Non-Existent Compounds, p. 162. New York: Marcel Dekker.

Dietzsch, W., Sieler, J., Meiler, W. \& Robien, W. (1998). Phosphorus, Sulfur Silicon Relat. Elem. 38, 293.

Duisenberg, A. J. M. (1992). J. Appl. Cryst. 25, 92-96.

Holmesland, O. \& Römming, C. (1966). Acta Chem. Scand. 20, 2601.

Hooft, R. W. W. (1998). Collect. Nonius BV, Delft, The Netherlands. Hope, H. \& McCullough, J. D. (1962). Acta Cryst. 15, 806-807.

Maddox, H. \& McCullough, J. D. (1966). Inorg. Chem. 5, 522-526.

Mont, W.-W. du, von Salzen, A. M., Ruthe, F., Seppala, E., Mugesh, G., Devillanova, F. A., Lippolis, V. \& Kuhn, N. (2001). J. Organomet. Chem. 623, 14-28.

Otwinowski, Z. \& Minor, W. (1997). Methods Enzymol. 276, 307326.

Pauling, L. (1960). The Nature of the Chemical Bond, 3rd ed. Ithaca, NY: Cornell University Press.

Sheldrick, G. M. (1990). Acta Cryst. A46, 467-473.

Sheldrick, G. M. (1998). SHELXL97-2. University of Göttingen, Göttingen, Germany.

Spek, A. L. (2003). J. Appl. Cryst. 36, 7-13.

Suwinska, K. (1995). Acta Cryst. B51, 248-254.

Timoshenko, V. M., Nikolin, Y. V., Chernega, A. N. \& Shermolovich, Y. G. (2002). Eur. J. Org. Chem. pp. 1619-1627. 